\title{
Dry Markets and Superreplication Bounds of American Derivatives
}

\author{
João Amaro de Matos ${ }^{1}$ and Ana Lacerda ${ }^{2}$ \\ Faculdade de Economia \\ Universidade Nova de Lisboa \\ Campus de Campolide \\ 1099-032 Lisbon, Portugal
}

October 13, 2004

\footnotetext{
${ }^{1}$ Corresponding author. Email: amatos@fe.unl.pt. Phone:+351962406397.

${ }^{2}$ Financial support from Fundação Amélia de Mello, INOVA and CMA-FCT is gratefully acknowledge.
} 


\begin{abstract}
This paper studies the impact of dry markets for underlying assets on the pricing of American derivatives, using a disrete time framework. Dry markets are characterized by the possibility of non-existence of trading at certain dates. Such non-existence may be deterministic or probabilistic. Using superreplicating strategies, we derive expectation representations for the range of arbitrage-free values of the dervatives. In the probabilistic case, if we consider an enlarged filtration induced by the price process and the market existence process, ordinary stopping times are required. If not, randomized stopping times are required. Several comparisons of the ranges obtained with the two market restrictions are performed. Finally, we conclude that arbitrage arguments are not enough to define the optimal exercise policy.
\end{abstract}

Keywords: American derivatives, pricing, incomplete markets, dry markets, superreplication, randomized stopping times, strong duality. 


\section{Introduction}

Among the traditional assumptions on which derivatives' pricing is based, markets are perfect and the underlying asset can be transacted at any point in time. Under the absence of arbitrage opportunities the value of a derivative can be computed as the value of a portfolio on the underlying risky asset and risk-free bonds that exactly replicates its payoff. Such portfolio can be rebalanced in a self-financing way until the maturity of the derivative, by continuously transacting the underlying asset and the bonds. Under these assumptions, the calculated value of the initial portfolio can be shown to be the equilibrium price of the derivative. Considering the case of American derivatives it has been shown by Bensoussan (1984) and Karatzas (1988) that, in this setting, the no-arbitrage value of one such derivative is indeed the supremum of the implied European derivative values over all possible stopping times.

In this paper we assume that an American derivative and its respective underlying asset cannot be transacted at some points in time, and study the impact of this constraint on the pricing of American derivatives. The fact that the assets can be transacted only at some points in time can be described as a lack of liquidity of the market, as in Longstaff (2001). We shall refer to this situation as dry markets. We will consider two different types of dry markets. In the first type, to be called deterministic illiquidity, we know ex-ante exactly at which points in time markets do exist or do not exist. In the second type, to be called probabilistic illiquidity, we assign a probability $p$ to the existence of the market at each point in time.

Markets' dryness implies that markets may become incomplete in the sense that perfect hedging of the derivative in all states of nature is no longer possible. However, for any given derivative, portfolios can be found that have the same payoff as the derivative in some states of nature and higher payoffs in the other states. Such portfolios are said to be superreplicating (or superhedging). Holding one such portfolio should be worth more than the derivative itself and therefore, the value of the cheapest of such portfolios should be seen as a bound on the value of the derivative. The nature of the superreplicating bounds for European derivatives is well characterized in the context of incomplete markets in the papers by El Karoui and Quenez $(1991,1995)$, Edirisinghe, Naik and Uppal (1993) and Karatzas and Kou (1996). A direct application to the case of European option pricing when the market for the underlying is dry can be found in Amaro de Matos and Antão (2001). As

all these results stress, under market incompleteness the hedging position of a market-maker is different depending on whether this intermediary is in a long or in a short position. This fact results in a lower and an upper bound 
for the derivatives' values.

The superreplicating bounds establish the limits of the interval for the prices outside which the market-maker has a positive profit with probability one. In other words, an arbitrage opportunity exists if the market-maker sells options above the upper bound or buys options below the lower bound.

There has been a relatively extensive literature in the continuous time setting, analyzing this problem and characterizing in varying degrees of generality the superhedging bounds of American derivatives in incomplete markets. Examples are the papers by Kramkov (1996), Follmer and Kramkov (1997), Follmer and Kabanov (1998) and Karatzas and Kou (1998). More recently, a paper by Jha and Chalasani (2001) discuss the particular case of transaction costs in discrete time and conclude that, in their specific setting, the superreplicating bounds of one such derivative may also be written as the supremum of the implied European derivative value. However, there are two important subtleties in their result: first, the supremum in this case must be taken over randomized stopping times and second, the probability measure defining the European value over which the supremum is taken, may depend itself on the randomized stopping time that solves the problem.

Jha and Chalasani (2001) relate their result to the fact that ${ }^{1}$, under incomplete markets, the choice of exercise policy may influence the characterization of the marketed subspace, and therefore influence the pricing of securities. A rational exercise policy may even not be well defined if the state-price deflator depends on the exercise policy. This argument would provide solid ground for the optimal randomized stopping times characterizing the superreplication bounds of the American derivatives under proportional transaction costs.

Our results show that, under dry markets, and in the same general discrete time setting used by Jha and Chalasani (2001), we can also write the superreplicating bounds of an American derivative as the supremum of the implied European derivative value. However, the supremum in this case may be taken over deterministic stopping times, as opposed to the intuition provided by the above cited authors. Although the result for deterministic illiquidity may be understood in the context of the superreplicating bounds discussed in Harrison and Kreps (1979), the case of probabilistic illiquidity is of a different nature since it crosses an additional source of uncertainty (existence or non existence of the market at a given point in time).

Our work is organized as follows. Section 2 models the case of Deterministic Illiquidity, introducing the model and relevant probabilistic concepts. Section 3 states the corresponding results, presenting the upper and lower

\footnotetext{
${ }^{1}$ For a discussion of this point see, among others, Duffie (2001), p.37.
} 
superreplicating bounds of American derivatives. This is followed by Section 4 that models the case of Probabilistic Illiquidity, after what Section 5 presents the corresponding results for the upper and lower superreplicating bounds of American derivatives. In section 6 these different bounds are compared. The exercised policy in dry markets is discussed in section 7. Finally, we section 8 we conclude. Our main technical proofs are presented in the Appendix.

\section{Deterministic Illiquidity}

\subsection{The model}

Consider an economy where three different assets are transacted. The first asset is a risk free asset with unitary initial value that provides a certain total return of $R$ per period; the second asset to be considered is a risky asset (the stock); finally, the third asset is an American derivative, written on the stock, with expiration date $T$. We work in discrete time, corresponding to dates $0,1, \ldots, T$. The set of these dates is denoted by $\mathcal{T} \equiv\{0,1, \ldots, T\}$. The evolution of the value of the underlying asset is modelled by means of a finite event tree. Each node of such tree is identified by a pair $(j, t)$, where $j$ denotes the $j$-th node at time $t$. There is only one node at time $t=0$, denoted by $(0,0)$. For any given node $(j, t)$, the set of successors at time $t+k, k>0$, is denoted by $j_{t}^{+}(t+k)$. For simplicity let $j_{t}^{+}$denote the set of immediate successors, i.e., $j_{t}^{+} \equiv j_{t}^{+}(t+1)$. The nodes $(j, T)$, at time $T$, are called terminal nodes and $j_{T}^{+}$is assumed to be the empty set $\emptyset$. It is also assumed that, for $t<T$, each nonterminal node $(j, t)$ has a nonempty set of immediate successors, i.e., $j_{t}^{+} \neq \emptyset$. In an analogous way, the set of immediate predecessors of a node $(j, t) \neq(0,0)$ is denoted by $j_{t}^{-}$. In what follows we shall consider the case where such sets $j_{t}^{-}$have a unique element. Moreover, we denote by $\mathcal{J}_{t}$ the set of all nodes at any point in time $t$

$$
\mathcal{J}_{t}=\cup_{j}(j, t) .
$$

A path on the event tree is a set of nodes $w=\cup_{t \in\{0,1, \ldots, T\}}\left(j_{t}, t\right)$ such that each element in the union satisfies $\left(j_{t+k}, t+k\right) \in j_{t}^{+}(t+k)$, with $k>0$ and $t+k \in\{0,1, \ldots, T\}$. Let $\Omega$ denote the set of all paths on the event tree. Each node in the tree represents the set of all tree paths that contain that node. Let $S$ denote the process followed by the stock price. More precisely, let $S(j, t)$ denote the price of the stock at node $(j, t)$. A natural filtration on the space $\Omega$ associated to the price process $S$ is $\mathcal{F}=\mathcal{F}_{0}, \mathcal{F}_{1}, \ldots, \mathcal{F}_{T}$, where each $\mathcal{F}_{t}$ is the $\sigma$-algebra generated by the random variable $S(\cdot, t)$. All the 
random variable will be defined in the measurable space $(\Omega, \mathcal{F})$. Similarly, let $G$ denote the process followed by the payoff of American derivative. Hence, $G(j, t)$ denotes the payoff of the American derivative at node $(j, t)$ whenever exercised at that point. Let $\bar{S}(j, t)$ and $\bar{G}(j, t)$ stand for the discounted values of the above processes, i.e.,

$$
\bar{G}(j, t)=\frac{G(j, t)}{R^{t}} \text { and } \bar{S}(j, t)=\frac{S(j, t)}{R^{t}} .
$$

Dry markets are characterized by the fact that transactions are possible only at some points in time. We hereby model dry markets allowing transactions only at times $t$ in a set $\mathcal{T}_{m} \subseteq \mathcal{T}$. It is also assumed that transactions are possible at times $t=0$ and $t=T$, i.e., $\{0, T\} \subseteq \mathcal{T}_{m}$.

At any node $(j, t)$ consider the portfolio constituted by $\Delta(j, t)$ shares of the underlying asset and an amount $B(j, t)$ invested in the risk free asset. One such portfolio is denoted by $[\Delta(j, t), B(j, t)]$. Its value process is given by

$$
V(j, t)=\Delta(j, t) S(j, t)+B(j, t) .
$$

Consider a short position on the American derivative. A replicating strategy is a sequence of portfolios $\{[\Delta(j, t), B(j, t)]\}_{t \in \mathcal{T}_{m}}$ such that the value of each of them is larger than or equal to the payoff of the derivative at any non-terminal node in the next transaction time. Additionally, at any terminal node its value is equal to the payoff of the derivative. In other words, for any two consecutive trading dates $t_{1}$ and $t_{2}>t_{1}$, consider an arbitrary node $\left(j, t_{1}\right)$ and the subset of its possible successors $j_{t_{1}}^{+}\left(t_{2}\right)$ Then, the portfolio at $t_{1},\left[\Delta\left(j, t_{1}\right), B\left(j, t_{1}\right)\right]$, must be such as to generate in $t_{2}$ a value $\Delta\left(j, t_{1}\right) S\left(i, t_{2}\right)+B\left(j, t_{1}\right) R^{t_{2}-t_{1}}$ such that

$$
\Delta\left(j, t_{1}\right) S\left(i, t_{2}\right)+B\left(j, t_{1}\right) R^{t_{2}-t_{1}} \geq G\left(i, t_{2}\right)
$$

with $\left(i, t_{2}\right) \in j_{t_{1}}^{+}\left(t_{2}\right)$ and if $t_{2}=T$ then

$$
\Delta\left(j, t_{1}\right) S(i, T)+B\left(j, t_{1}\right) R^{T-t_{1}}=G(i, T) .
$$

A self-financed portfolio is a portfolio that generates enough wealth to rebalance the portfolio according to any future state of nature. In other words, for any two consecutive trading dates $t_{1}$ and $t_{2}>t_{1}$, consider an arbitrary node $\left(j, t_{1}\right)$ and the set of its possible successors $\left\{\left(i, t_{2}\right): i \in j_{t_{1}}^{+}\right\}$. Then, the value of the portfolio at that point in time, $\Delta\left(j, t_{1}\right) S\left(j, t_{1}\right)+B\left(j, t_{1}\right)$ must be such as to generate

$$
\Delta\left(j, t_{1}\right) S\left(i, t_{2}\right)+B\left(j, t_{1}\right) R^{t_{2}-t_{1}} \geq V\left(i, t_{2}\right) .
$$


For a long position on the American derivative, analogous definitions are obtained with reverted inequalities, but only for the nodes $(i, t)$ such that the option would be exercised, i.e., such that $G(i, t)>V(i, t)$.

If a complete market is considered the value of an American derivative is the value of the cheapest self-financing portfolio on the underlying risky asset and risk-free bonds that replicates the payoff of the American derivative. For this portfolio the following condition will hold at any non-terminal node

$$
\Delta\left(j, t_{1}\right) S\left(i, t_{2}\right)+B\left(j, t_{1}\right) R^{t_{2}-t_{1}}=\max \left[V\left(i, t_{2}\right), G\left(i, t_{2}\right)\right] .
$$

In dry markets, however, the number of transacted securities may be insufficient to allow the construction of a self-financing portfolio that replicates the payoff of an American derivative. In other words, markets may become incomplete. In that case, there is not a unique arbitrage free value for the American derivative. However, replacing the notion of replicating strategy by the notion of superreplication strategy it is possible to derive an arbitrage free range of variation for the value of the American derivative. In order to find the upper bound of this range consider a short position in the derivative. The upper bound will be the value of the cheapest portfolio that the buyer of the derivative can buy in order to completely hedge against any possibility of exercise of the American derivative and without need of additional financing at any rebalancing dates. Note that in order to completely hedge against the possibility of exercise the value of the portfolio, at any given node, has to be equal or higher than the payoff of the American derivative. In that case it is said that the portfolio superreplicates the payoff of the American derivative. On the other hand, in order to find the lower bound of the arbitrage-free range of variation consider a long position in the derivative. For a given exercise policy consider the most expensive portfolio that the buyer of the American derivative can buy in order to be fully hedged. The lower bound is the value of the most expensive portfolio chosen among the portfolio just described. Note that in this case the buyer of the American derivative is, for a given exercise policy, completely hedged if in any node where the option may be exercised the payoff of the American derivative is higher than the value of the hedging portfolio. In this case it is said that the portfolio is superreplicated by the American derivative.

Under market completeness, both limiting portfolios coincide with a replicating portfolio and the value of the derivative is well characterized [Karatzas (1988)]. Under market incompleteness however, that is no longer true and the arbitrage-free value of the derivative must lie between the values of the two limiting superreplicating portfolios.

In what follows we are going to characterize the upper and lower arbitragefree bounds for the value of the American derivatives in the framework de- 
scribed above. In order to do that, we first define some mathematical objects, such as node probability measure, adjusted probability measure and stopping time.

\subsection{Some Probabilistic Definitions}

Definition 2.1 A node probability measure is a nonnegative node function $q(j, t)$ such that

$$
\sum_{t \in \mathcal{T}_{m}} \sum_{(j, t) \in \mathcal{J}_{t}} q(j, t)=1 .
$$

The set of all node probability measures is denoted by $Q$.

Definition 2.2 A node probability measure on the event tree is said to be simple if, for $t \in \mathcal{T}_{m}$ and $t+k \in \mathcal{T}_{m}$, there are no two nodes in the same path, say $(i, t)$ and $(j, t+k) \in i_{t}^{+}(t+k)$, such that $q(i, t)>0$ and $q(j, t+k)>0$.

The following theorem is analogous to theorem 6.7 of Jha and Chalasani (2001) but now in the framework of dry markets.

Theorem 2.1 (Jha and Chalasani) The extreme points of the set of nodes $Q$ are simple node probability measures, i.e., on every path on the event tree there is at most one node where $q$ is strictly positive.

The proof of this theorem follows closely the proof of theorem 6.7 in Jha and Chalasani (2001) and is presented in the appendix A.

Definition 2.3 An adjusted probability measure is a nonnegative function $P(i, t)$ such that $P(0,0)=1$ and for all $t \in \mathcal{T}_{m}$

$$
P(i, t)=\sum_{(j, s) \in i^{+}(s)} P(j, s)
$$

with $s=\min \left\{z \in \mathcal{T}_{m}: z>t\right\}$.

The set of all probability measures is denoted by $\mathbb{P}$.

Definition 2.4 A process $Z=\left\{Z_{t}: t \in \mathcal{T}_{m}\right\}$ is called adapted to the filtration $\mathcal{F}$ if for each $t \in \mathcal{T}_{m}, Z_{t}$ is $\mathcal{F}_{t}$-measurable. 
Let $\tau$ denote an ordinary stopping time that takes values in $\mathcal{T}_{m}$, i.e., $\tau$ is a map such that $\tau: \Omega \rightarrow \mathcal{T}_{m}$ and $\{w: \tau(w) \leq t\} \in \mathcal{F}_{t}$ for all $t \in \mathcal{T}_{m}$. We define a nonnegative adapted process $X_{\tau}$ associated with $\tau$ that is defined for all $t \in \mathcal{T}_{m}$ and has the form $X_{\tau}(i, k)=1$ if $\tau(w)=k$ and $X_{\tau}(i, k)=0$ otherwise, where $(i, k)$ is a node in path $w$. Let $\mathbb{T}$ and $\mathbb{X}_{\mathbb{T}}$ denote the set of all $\tau$ and associated $X_{\tau}$, respectively.

Definition 2.5 A simple node probability measure is said to be associated with a given stopping time if at any node such that $X_{\tau}(i, k)$ is equal to zero then $q(i, t)$ is also equal to zero. Moreover, at any node such that $X_{\tau}(i, k)$ is strictly positive then $q(i, t)$ is also positive.

The set of all node probability measures with this property is denoted by $Q^{\tau}$.

Definition 2.6 For any adjusted probability measure $P \in \mathbb{P}$ and stopping time $\tau \in \mathbb{T}$ we say that $P$ is a $\tau$-martingale measure if, $P$-almost surely, for any $(i, t)$ with $t \in \mathcal{T}_{m}$ we have

$$
\sum_{m>t, m \in \mathcal{T}_{m}} \sum_{(j, m) \in i_{t}^{+}(m)} p(j, m) X_{\tau}(j, m)[\bar{S}(i, t)-\bar{S}(j, m)]=0
$$

The set of all $P$ that have this property is denoted by $\mathcal{P}(\tau)$.

Definition 2.7 For any adjusted probability measure $P \in \mathbb{P}$ we say that $P$ is a martingale measure if, $P$-almost surely, for any $(i, t) \in \mathcal{J}_{t}$ with $t \in \mathcal{T}_{m}$ we have

$$
\sum_{m>t, m \in \mathcal{T}_{m}} \sum_{(j, m) \in i_{t}^{+}(m)} p(j, m)[\bar{S}(i, t)-\bar{S}(j, m)]=0
$$

The set of all $P$ that have this property is denoted by $\mathcal{P}$.

Let $\left(P, X_{\tau}\right)$ denote a measure-strategy pair, i.e., a pair constituted by an adjusted probability measure and a nonnegative adapted process.

Definition 2.8 A measure-strategy pair $\left(P, X_{\tau}\right)$ is said to be equivalent to a node probability measure if $P(i, t) X_{\tau}(i, t)=q(i, t)$ for any given node $(i, t)$

We can now enunciate a fundamental result. 
Theorem 2.2 (Jha and Chalasani) Let $\left(P, X_{\tau}\right)$ be a measure-strategy pair. The simple node function $q$ defined by $q(i, t)=P(i, t) X_{\tau}(i, t)$ is the unique equivalent node-measure. Conversely, for a given simple node probability measure, $q$, there is a measure-strategy pair $\left(P, X_{\tau}\right)$ equivalent to $q$, where $P$ is uniquely defined at nodes $(i, t)$ where $q(i, t)+\sum_{\substack{(j, \tau) \in i_{t}^{+}(\tau) \\ \tau>t, \tau \in \mathcal{T}_{m}}} q(j, \tau)>0$, and $X_{\tau}$ is uniquely defined at nodes $(i, t)$ where $q(i, t)>0$.

A version of the proof of this result, adjusted to case of dry markets, is provided in Appendix A.

\section{Results on Deterministic Illiquidity}

\subsection{Upper bound for the Value of an American Deriva- tive}

The upper bound for the value of an American derivative is the maximum value for which the derivative would be transacted without allowing for arbitrage opportunities. In order to find the upper bound consider a short position in the derivative. The maximum value for which the derivative would be transacted without allowing for arbitrage opportunities would be the value of the cheapest portfolio that the buyer of the derivative can buy in order to completely hedge against any possibility of exercise of the American derivative and without need of additional financing at any rebalancing dates. A portfolio is initially built such that, at each transaction date until maturity, it generates enough wealth, so as to be rebalanced according to any revealed state of nature. Since by construction there is no need of additional financing, one such strategy is said to be a self-financed strategy. Additionally, it has to be a superreplicating strategy, i.e., a sequence of portfolios $\{[\Delta(j, t), B(j, t)]\}_{t \in \mathcal{I}_{m}}$ such that their values are greater or equal to the payoff of the derivative at any node in the next transaction time. In other words, for any two consecutive trading dates $t_{1}$ and $t_{2}>t_{1}$, consider an arbitrary node $\left(j, t_{1}\right)$ and the subset of its possible successors $j_{t_{1}}^{+}\left(t_{2}\right)$. Then, the portfolio at $t_{1},\left[\Delta\left(j, t_{1}\right), B\left(j, t_{1}\right)\right]$, must be such as to generate in $t_{2}$ a value $\Delta\left(j, t_{1}\right) S\left(i, t_{2}\right)+B\left(j, t_{1}\right) R^{t_{2}-t_{1}}$ such that

$$
\Delta\left(j, t_{1}\right) S\left(i, t_{2}\right)+B\left(j, t_{1}\right) R^{t_{2}-t_{1}} \geq G\left(i, t_{2}\right),
$$

with $\left(i, t_{2}\right) \in j_{t_{1}}^{+}\left(t_{2}\right)$.

More formally, take any $t_{1} \in \mathcal{T}_{m}$, such that $t_{1} \neq T$. Define the consecutive trading date as $t_{2}=\min \left(s \in \mathcal{T}_{m}: s>t_{1}\right)$. The upper bound for the value 
of the American derivative can thus be seen as the solution of the following problem:

$$
V_{d}^{u}=\min _{\{\Delta(j, t), B(j, t)\}} \Delta(0,0) S(0,0)+B(0,0)
$$

subject to the superreplicating restrictions:

$$
\begin{gathered}
\Delta(0,0) S(0,0)+B(0,0) \geq G(0,0), \\
\Delta\left(j, t_{1}\right) S\left(i, t_{2}\right)+B\left(j, t_{1}\right) R^{t_{2}-t_{1}} \geq G\left(i, t_{2}\right),
\end{gathered}
$$

for all $t_{1} \in \mathcal{T}_{m} \backslash\{T\}$ and $\left(i, t_{2}\right) \in j^{+}\left(t_{2}\right)$ with $t_{2}=\min \left(s \in \mathcal{T}_{m}: s>t_{1}\right)$ and subject to the self-financing restrictions:

$$
\Delta\left(j, t_{1}\right) S\left(i, t_{2}\right)+B\left(j, t_{1}\right) R^{t_{2}-t_{1}} \geq V\left(i, t_{2}\right)
$$

for all $t_{1} \in \mathcal{T}_{m} \backslash\left\{\max \left\{t \in \mathcal{T}_{m}: t<T\right\}, T\right\}$ and $\left(i, t_{2}\right) \in j^{+}\left(t_{2}\right)$ with $t_{2}=$ $\min \left(s \in \mathcal{T}_{m} \backslash\{T\}: s>t_{1}\right)$.

Using results from linear programming the upper bound arbitrage free bound of the American derivative can be written as follows.

Theorem 3.1 There exists a node probability measure $q \in Q$ such that the upper hedging price of an American derivative in a dry market can be written as

$$
V_{d}^{u}=\max _{q \in Q} \sum_{t \in \mathcal{T}_{m}} \sum_{(j, t)} q(j, t) \bar{G}(j, t)
$$

such that for any $(i, t)$ with $t \in \mathcal{T}_{m}$

$$
\sum_{m>t, m \in \mathcal{T}_{m}} \sum_{(j, m) \in i_{t}^{+}(m)} q(j, m)[\bar{S}(i, t)-\bar{S}(j, m)]=0 .
$$

Proof. As the problem that must solved in order to find the upper bound of the American derivative is a linear programming problem it is possible to construct its dual. Let $\lambda(0,0), \lambda\left(i, t_{2}\right)$ and $\alpha\left(i, t_{2}\right)$ be the dual variables associated with restrictions (2), (3) and (4), respectively. Then, the dual problem is

$$
\max \sum_{t \in \mathcal{T}_{m}} \lambda(j, t) S(j, t)
$$


subject to

$$
\begin{gathered}
\lambda(0,0) S(0,0)+\sum_{(i, t) \in i_{0}^{+}(t)}[\lambda(i, t)+\alpha(i, t)] S(i, t)=S(0,0) \\
\lambda(0,0)+\sum_{(i, t) \in i_{0}^{+}(t)}[\lambda(i, t)+\alpha(i, t)] R^{t}=1
\end{gathered}
$$

with $t=\min \left(s \in \mathcal{T}_{m}: s>0\right)$,

$$
\begin{gathered}
\sum_{\left(j, t_{2}\right) \in i_{t_{1}}^{+}\left(t_{2}\right)} S\left(j, t_{2}\right)\left[\lambda\left(j, t_{2}\right)+\alpha\left(j, t_{2}\right)\right]-\alpha\left(i, t_{1}\right) S\left(i, t_{1}\right)=0 \\
\sum_{\left(j, t_{2}\right) \in i_{t_{1}}^{+}\left(t_{2}\right)}\left[\lambda\left(j, t_{2}\right)+\alpha\left(j, t_{2}\right)\right] R^{t_{2}-t_{1}}-\alpha\left(i, t_{1}\right)=0
\end{gathered}
$$

for all $t_{1} \in T \backslash\left\{0, \max \left\{s \in \mathcal{T}_{m}: s<T\right\}, T\right\}$ and $t_{2}=\min \left(s \in \mathcal{T}_{m}: s>t_{1}\right)$, and, finally,

$$
\begin{gathered}
\sum_{(j, T) \in i_{t}^{+}(T)} S(j, T) \lambda(j, T)-\alpha(i, t) S(i, t)=0 \\
\sum_{(j, T) \in i^{+}(T)} S(j, T) R^{T-t}-\alpha(i, t)=0
\end{gathered}
$$

for all $t=\max \left\{s \in \mathcal{T}_{m}: s<T\right\}$.

Note that the restrictions (5), (7) and (9) of the dual problem are associated with the variables $\Delta(0,0), \Delta\left(i, t_{1}\right)$ and $\Delta(i, t)$, respectively, of the primal problem. In a similar way, the restrictions (6), (8) and (10) are, respectively, associated with the primal variables $B(0,0), B\left(i, t_{1}\right)$ and $B(i, t)$.

The restrictions presented in equations (7) and (9) can be rewritten such that, for all $t \in \mathcal{T}_{m} \backslash T$, we have

$$
\sum_{\substack{(j, m) \in i_{t}^{+}(m) \\ m>t, m \in \mathcal{T}_{m}}} \lambda(j, m) R^{m-t} S(i, t)=\sum_{\substack{(j, m) \in i^{+}(m) \\ m>t, m \in \mathcal{T}_{m}}} \lambda(j, m) S(j, m)
$$

From equations (6), (8) and (10) we obtain

$$
\sum_{\substack{(i, t) \\ t \in \mathcal{T}_{m}}} \lambda(i, t) R^{t}=1
$$

Considering equations (12), (5) and (12), we have, for all $(i, t)$,

$$
\sum_{\substack{(j, m) \in i^{+}{ }_{m>t}(m) \\ m>m \in \mathcal{T}_{m}}} \lambda(j, m) R^{m-t} S(i, t)=\sum_{\substack{(j, m) \in i^{+}(m) \\ m>t, m \in \mathcal{T}_{m}}} \lambda(j, m) S(j, m) .
$$


Let $q(i, t)=\lambda(i, t) R^{t}$. For any $t \in \mathcal{I}_{m} \backslash\{T\}$,

$$
\sum_{\substack{(j, m) \in i^{+}(m) \\ m>t, m \in \mathcal{T}}} q(j, m) \bar{S}(i, t)=\sum_{\substack{(j, m) \in i^{+}(m) \\ m>t, m \in \mathcal{T}}} q(j, m) \bar{S}(j, m) .
$$

Hence, the dual problem can be written as

$$
\max _{q \in Q} \sum_{\substack{(i, t) \in \mathcal{J}_{t} \\ t \in \mathcal{T}_{m}}} q(i, t) \bar{G}(i, t)
$$

such that for any $(i, t)$ with $t \in \mathcal{T}_{m}$

$$
\sum_{m>t, m \in \mathcal{T}_{m}} \sum_{(j, m) \in i_{t}^{+}(m)} q(j, m)[\bar{S}(i, t)-\bar{S}(j, m)]=0 .
$$

The upper bound solving the problem above can also be seen as the solution of a more intuitive problem. In fact, it can be shown that this upper bound maximizes over all possible stopping times the expected discounted payoff, when the expectation is optimized among all adjusted probability measures. In other words,

Theorem 3.2 There exists an adjusted probability measure $P \in \mathcal{P}(\tau)$ and an adapted process $X_{\tau} \in \mathbb{X}_{\mathbb{T}}$ such that the upper hedging price of an American derivative in a dry market can be written as

$$
V_{d}^{u}=\max _{\tau \in \mathbb{T}} \max _{P \in \mathcal{P}(\tau)} E^{p} G_{\tau}
$$

with $G_{\tau}(i, t)=X_{\tau}(i, t) G(i, t)$. Additionally, if there is a probability measure with positive probability on every path then the upper hedging price of an American derivative in a dry market can be rewritten as

$$
V_{d}^{u}=\max _{\tau \in \mathbb{T}} \max _{P \in \mathcal{P}} E^{p} G_{\tau}
$$

where $G_{\tau}(i, t)=X_{\tau}(i, t) G(i, t)$, as before.

Proof. As $Q$ is a convex set, the maximum of the problem

$$
\max _{q \in Q} \sum_{\substack{(i, t) \in \mathcal{J}_{t} \\ t \in \mathcal{T}_{m}}} q(i, t) \bar{G}(i, t)
$$

such that for any $(i, t)$ with $t \in \mathcal{T}_{m}$

$$
\sum_{m>t, m \in \mathcal{T}_{m}} \sum_{(j, m) \in i_{t}^{+}(m)} q(j, m)[\bar{S}(i, t)-\bar{S}(j, m)]=0 .
$$


is obtained at the extremes points of $Q$.

By theorem (2.1) we know that the extremes points are simple node measures. Using theorem (2.2) we can rewrite the problem above as

$$
\max _{\tau \in \mathbb{T}} \max _{P \in \mathcal{P}(\tau)} E^{p} \bar{G}_{\tau}
$$

where

$$
\bar{G}_{\tau}(i, t)=\bar{G}(i, t) X_{\tau}(i, t) .
$$

As stressed in Jha and Chalasani (2001), page 64, if there is a martingale measure $\hat{P} \in \mathcal{P}$ with positive measure on every path, $w$, the inner maximization in (13) can be restricted to all $P \in \mathcal{P}$ without affecting its value. First, any $P \in \mathcal{P}$ also belongs to $\mathcal{P}(\tau)$. Second, any measure $P \in \mathcal{P}$ can be redefined to be a martingale measure $P^{\prime} \in \mathcal{P}(\tau)$ such that $E^{P^{\prime}} \bar{G}_{\tau}=E^{P} \bar{G}_{\tau}$, as follows

$$
P^{\prime}(i, t)= \begin{cases}P(i, t), & t \leq k: \text { if }(i, t) \in w \text { and } \tau(w)=k \\ P_{-}^{\prime}(i, t) \frac{\hat{P}(i, t)}{\hat{P}_{-}(i, t)} & \text { otherwise }\end{cases}
$$

where $P_{-}^{\prime}(i, t)$ and $\hat{P}_{-}(i, t)$ are the probabilities in the node that is an immediate predecessor of $(i, t)$.

\subsection{Lower bound for the Value of an American Deriva- tive}

The lower bound for the value of an American derivative is the minimum value for which the derivative would be transacted without allowing for arbitrage opportunities. In order to find the lower bound consider a long position in the derivative. As stressed in Karatzas and Kou (1998) while the seller of the American derivative has to be hedged against any possible exercise policy, the buyer of the American derivative needs only to hedge against a given exercise policy that is defined by himself. For a given exercise policy consider the most expensive portfolio that the buyer of the American derivative can buy in order to be fully hedged and without need of additional financing at any rebalancing dates. The minimum value for which the derivative would be transacted without allowing for arbitrage opportunities would be the value of most expensive portfolio chosen among all the portfolios just mentioned.

For any given exercise policy $\tau$ and any node $(j, t)$, such that $(j, t)$ is before the exercise time, consider the portfolio constituted of $\Delta^{\tau}(j, t)$ shares of the underlying asset and an amount $B^{\tau}(j, t)$ invested in the risk free asset. 
For each exercising policy we are looking for the most expensive portfolio that the buyer of the American derivative can buy that is self-financed and is supperreplicated by the payoffs of the American derivative. A portfolio $\left[\Delta^{\tau}\left(j, t_{1}\right), B^{\tau}\left(j, t_{1}\right)\right]$ is said self-financing if, for any two consecutive trading dates $t_{1}$ and $t_{2}>t_{1}$ and an arbitrary node $\left(j, t_{1}\right)$, the portfolio is such as to generate in $t_{2}$ a value $\Delta^{\tau}\left(j, t_{1}\right) S\left(i, t_{2}\right)+B^{\tau}\left(j, t_{1}\right) R^{t_{2}-t_{1}}$ such that

$$
\Delta^{\tau}\left(j, t_{1}\right) S\left(i, t_{2}\right)+B^{\tau}\left(j, t_{1}\right) R^{t_{2}-t_{1}} \leq V^{\tau}\left(i, t_{2}\right),
$$

for any node $\left(i, t_{2}\right) \in j_{t_{1}}^{+}\left(t_{2}\right)$ before the exercise of the American derivative. Additionally, a portfolio $\left[\Delta^{\tau}\left(j, t_{1}\right), B^{\tau}\left(j, t_{1}\right)\right]$ is said to be superreplicated by $G_{\tau}\left(i, t_{2}\right)$ if, for any two consecutive trading dates $t_{1}$ and $t_{2}>t_{1}$ and an arbitrary node $\left(j, t_{1}\right)$, it generates in $t_{2}$ a value $\Delta^{\tau}\left(j, t_{1}\right) S\left(i, t_{2}\right)+B^{\tau}\left(j, t_{1}\right) R^{t_{2}-t_{1}}$ such that

$$
\Delta^{\tau}\left(j, t_{1}\right) S\left(i, t_{2}\right)+B^{\tau}\left(j, t_{1}\right) R^{t_{2}-t_{1}} \leq G_{\tau}\left(i, t_{2}\right)
$$

for any $\left(i, t_{2}\right) \in j_{t_{1}}^{+}\left(t_{2}\right)$ when it is optimal to the holder of the American option to exercise it given $\tau$. The minimum value for which the derivative would be transacted without allowing for arbitrage opportunities would be the value of the most expensive portfolio chosen among all stopping times.

The lower bound for the value of the American derivative can thus be seen as the solution of the following problem:

$$
V_{d}^{l}=\max _{\tau \in \mathbb{T}} \max _{\left\{\Delta^{\tau}(j, t), B^{\tau}(j, t)\right\}} \Delta_{\tau}(0,0) S(0,0)+B_{\tau}(0,0)
$$

subject to the superreplicating restriction

$$
\Delta_{\tau}(0,0) S(0,0)+B_{\tau}(0,0) \leq G_{\tau}(0,0)
$$

if $\tau(w)=0$ and, otherwise,

$$
\Delta_{\tau}\left(j, t_{1}\right) S\left(m, t_{3}\right)+B_{\tau}\left(j, t_{1}\right) R^{t_{3}-t_{1}} \leq G_{\tau}\left(m, t_{3}\right)
$$

for any $\left(i, t_{2}\right)$ such that $X_{\tau}\left(m, t_{3}\right)=1$ and to the self-financing restrictions:

$$
\Delta_{\tau}\left(j, t_{1}\right) S\left(i, t_{2}\right)+B_{\tau}\left(j, t_{1}\right) R^{t_{2}-t_{1}} \leq \Delta_{\tau}\left(i, t_{2}\right) S\left(i, t_{2}\right)+B_{\tau}\left(i, t_{2}\right)
$$

for any $t_{1}$ with $\left(i, t_{2}\right) \in j_{t_{1}}^{+}\left(t_{2}\right)$ such that $X_{\tau}\left(i, t_{2}\right)=0$ and $X_{\tau}\left(j, t_{3}\right)=1$ for some $\left(j, t_{3}\right) \in j_{t_{2}}^{+}\left(t_{3}\right)$.

Using results from linear programming the upper bound arbitrage free bound of the American derivative can be written as follows. 
Theorem 3.3 There exists a node probability measure $q \in Q^{\tau}$ and a stopping time $\tau \in \mathbb{T}$ such that the upper hedging price of an American derivative in a dry market can be written as

$$
V_{d}^{l}=\max _{\tau \in \mathbb{T}} \min _{q \in Q^{\tau}} \sum_{(j, t)} \sum_{t \in \mathcal{T}_{m}} q(j, t) \bar{G}_{\tau}(j, t)
$$

with $\bar{G}_{\tau}(j, t)=\bar{G}(j, t) X_{\tau}(j, t)$ and for any $(i, t)$ and $t \in \mathcal{T}_{m}$

$$
\sum_{m>t, m \in \mathcal{T}_{m}} \sum_{(j, m) \in i_{t}^{+}(m)} q(j, m)[\bar{S}(i, t)-\bar{S}(j, m)]=0 .
$$

Proof. For a given stopping time the problem that must be solved in order to find the lower bound for the value of the American derivative is

$$
\max _{\{\Delta(j, t), B(j, t)\}} \Delta_{\substack{(j, t) \in \mathcal{J}_{t} \\ t \in \mathcal{T} \backslash\{T\}}} \Delta_{\tau}(0,0) S(0,0)+B_{\tau}(0,0)
$$

subject to the following superrepilcating conditions

$$
\Delta_{\tau}(0,0) S(0,0)+B_{\tau}(0,0) \leq G_{\tau}(0,0)
$$

if $X_{\tau}(0,0)=1$, and,

$$
\Delta_{\tau}\left(j, t_{1}\right) S\left(i, t_{2}\right)+B\left(j, t_{1}\right) R^{t_{2}-t_{1}} \leq G_{\tau}\left(i, t_{2}\right),
$$

for all $t_{1} \in \mathcal{T}_{m} \backslash\{T\}$ and $\left(i, t_{2}\right) \in j^{+}\left(t_{2}\right)$ with $t_{2}=\min \left(s \in \mathcal{T}_{m}: s>t_{1}\right)$, if $X_{\tau}\left(i, t_{2}\right)=1$.

Additionally, for any node $\left(i, t_{2}\right)$ such that $X_{\tau}\left(i, t_{2}\right)=1=1$ the selffinancing conditions apply, i.e.,

$$
\Delta_{\tau}\left(k, t_{1}\right) S\left(i, t_{2}\right)+B_{\tau}\left(k, t_{1}\right) R^{t_{2}-t_{1}} \leq \Delta_{\tau}\left(i, t_{2}\right) S\left(i, t_{2}\right)+B\left(i, t_{2}\right)
$$

for all $t_{1} \in \mathcal{T}_{m} \backslash\left\{\max \left\{t \in \mathcal{T}_{m}: t<T\right\}, T\right\}$ and $\left(i, t_{2}\right) \in j^{+}\left(t_{2}\right)$ with $t_{2}=$ $\min \left(s \in \mathcal{T}_{m} \backslash\{T\}: s>t_{1}\right)$.

Using an analogous procedure as in the proof where the upper bound for the value of the American derivative was found we can write the dual problem of the linear optimization problem described above

$$
\min _{q \in Q^{\tau}} \sum_{\substack{(i, t) \in \mathcal{J}_{t} \\ t \in \mathcal{T}_{m}}} q(i, t) \bar{G}_{\tau}(i, t)
$$

such that for any $(i, t)$ with $t \in \mathcal{T}_{m}$

$$
\sum_{m>t, m \in \mathcal{T}_{m}} \sum_{(j, m) \in i_{t}^{+}(m)} q(j, m)[\bar{S}(i, t)-\bar{S}(j, m)]=0 .
$$


Optimizing with relation to $\tau$ the problem becomes

$$
\max _{\tau \in \mathbb{T}} \min _{q \in Q^{\tau}} \sum_{\substack{(i, t) \in \mathcal{J}_{t} \\ t \in \mathcal{T}_{m}}} q(i, t) \bar{G}_{\tau}(i, t)
$$

such that for any $(i, t)$ with $t \in \mathcal{T}_{m}$

$$
\sum_{m>t, m \in \mathcal{T}_{m}} \sum_{(j, m) \in i_{t}^{+}(m)} q(j, m)[\bar{S}(i, t)-\bar{S}(j, m)]=0 .
$$

Theorem 3.4 There exists an adjusted probability measure $P \in \mathcal{P}(\tau)$ and a stopping time $\tau \in \mathbb{T}$ such that the upper hedging price of an American derivative in a dry market can be written as

$$
V_{d}^{l}=\max _{\tau \in \mathbb{T}} \min _{P \in \mathcal{P}(\tau)} E_{\tau}^{p} \bar{G}_{\tau}
$$

with $G_{\tau}(i, t)=X_{\tau}(i, t) G(i, t)$. Additionally, if there is a probability measure with positive probability on every path then the upper hedging price of an American derivative in a dry market can be rewritten as

$$
V_{d}^{l}=\max _{\tau \in \mathbb{T}} \min _{P \in \mathcal{P}} E^{p} G_{\tau}
$$

where $G_{\tau}(i, t)=X_{\tau}(i, t) G(i, t)$, as before.

Proof. Using the result presented in theorem (3.3) and the theorem (2.2) the proof is straightforward.

This resulted has already been conjectured as an extension in Harrison and Kreps (1979). When the market is complete then $\mathcal{P}$ is a singleton and the two bounds coincide with the unique arbitrage free value of the American derivative.

In the following section the upper and lower upper arbitrage free bound of the American derivatives when the illiquidity is not deterministic but probabilistic. At certain dates there is uncertainty about the existence of the market.

\section{Probabilistic Illiquidity}

\subsection{The model}

As in the previous section we shall work in discrete time, corresponding to dates in $\mathcal{T}=\{0,1, \ldots, T\}$. Let $\mathcal{T}_{m} \subseteq \mathcal{T}$ be a set of points in time such 
that transactions are possible with probability one for all times $t \in \mathcal{T}_{m}$. By assumption, both 0 and $T$ belong to $\mathcal{T}_{m}$, i.e., transactions are certainly possible at times $t=0$ and $t=T$. Similarly, let $\mathcal{T}_{p} \subseteq \mathcal{T}$ be defined as the set of points in time such that transactions are possible, but not certain. For each time $t \in \mathcal{T}_{p}$, we assume that transactions are possible with an exogenous probability $p>0$ with $\mathcal{T}_{m} \cup \mathcal{T}_{p}=\mathcal{T}$ and $\mathcal{T}_{m} \cap \mathcal{T}_{p}=\emptyset$

We can think of the existence (or not) of the market at time $t$ as the realization of a random variable $y_{t}$. This random variable is defined for all $t \in \mathcal{T}$ and it is assumed to be independent of the ordinary source of uncertainty that generates the price process. We can therefore talk about a market existence process. In order to construct one such process, let us first start with the state space. Let $\#\left(\mathcal{T}_{p}\right)$ denote the number of points in $\mathcal{T}_{p}$. At each of these points, market may either exist or not exist, leading to $2^{\#\left(\mathcal{T}_{p}\right)}$ possible states of nature. We then have the collection of possible

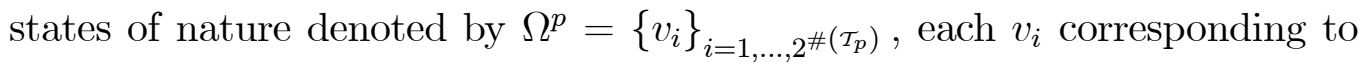
a distinct state. Recall that $\Omega$ denotes the set of paths $(w)$ in a perfectly liquid market and $\mathcal{F}_{t}$ is the $\sigma$-algebra generated by the random variable $S_{t}$. We now consider the new extended measurable space $(\bar{\Omega}, \overline{\mathcal{F}})$, where

$$
\bar{\Omega}=\Omega \times \Omega^{p}
$$

and

$$
\overline{\mathcal{F}}=\mathcal{F} \times \mathcal{F}^{p},
$$

with $\mathcal{F}^{p}=\mathcal{F}_{0}^{p}, \mathcal{F}_{1}^{p}, \ldots, \mathcal{F}_{T}^{p}$, where $\mathcal{F}_{t}^{p}$ is the $\sigma$-algebra generated by the random variable $y_{t}$. Also recall that the random variable $y_{t}$ assumes the values 0 (when there is no market) and 1 (when there is market) and is not dependent on $w$. Note also that the variable $y_{t}$ depends only on the information in $\mathcal{F}_{p}$. Let $p_{y}$ be the probability associated with the random variable $y_{t}$. For all $t \in \mathcal{T}_{p}$, we have $p_{y}\left(y_{t}=1\right)=p$ and $p_{y}\left(y_{t}=0\right)=1-p$. Similarly, for all $t \in \mathcal{T}_{m}, p_{y}\left(y_{t}=1\right)=1$ and $p_{y}\left(y_{t}=0\right)=0$. Let the $T+1$ dimensional vector $\mathbf{y}$ denote a given realization of the process $\left\{y_{t}\right\}_{t \in \mathcal{T}}$. There are $2^{\#\left(\mathcal{T}_{p}\right)}$ different possible vectors $\mathbf{y}$.

As in section 2.1, the process followed by the stock price is denoted by $S$. However, in the presence of probabilistic dry markets the stock price is only observed when market exists, i.e., in all nodes $(i, t)$ such that $y(i, t)=1$.

As a motivation to what follows, let us consider an example. Consider $\mathcal{T}_{m}=\{0,2,4\}$ and $\mathcal{T}_{p}=\{1,3\}$. At $t=1$ there is a $(1-p)$ chance that the stock price will not be observed. The same thing happens at $t=3$. Hence, if there is no new information at these points in time, the $\sigma$-algebra describing the information available to the market will be $\mathcal{F}_{t}=\mathcal{F}_{t-1}$. In our 
example, there are four different vectors $\mathbf{y}$, given by $\mathbf{y}_{1}=(1,1,1,1,1), \mathbf{y}_{2}=$ $(1,0,1,1,1), \mathbf{y}_{3}=(1,1,1,0,1)$ and $\mathbf{y}_{4}=(1,0,1,0,1)$. Each one is associated with a given probability, respectively, $p^{2}, p(1-p), p(1-p)$ and $(1-p)^{2}$. We may describe the trees of information process associated to each of the four possible circumstances as follows

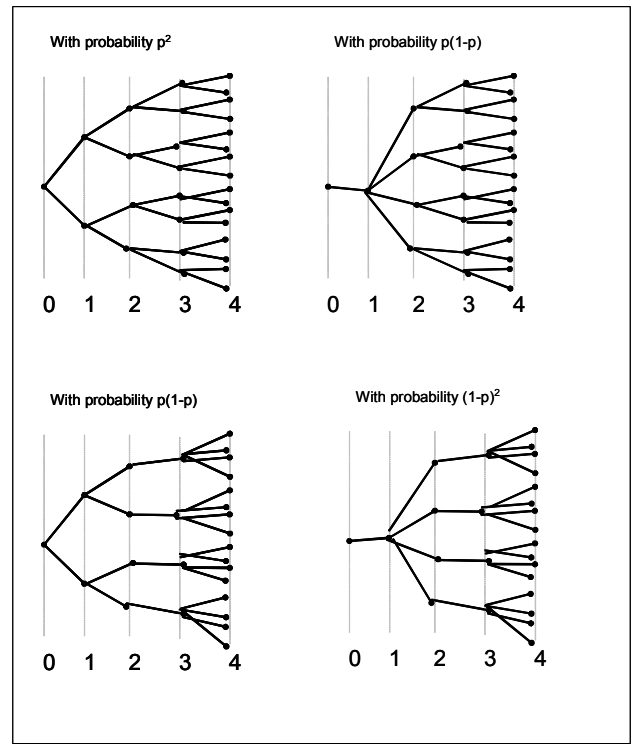

Figure 1: For each $\mathbf{y}$ the information available to the market can be represented by a different tree.

The first tree (top, left) describes the case corresponding to vector $\mathbf{y}_{1}$, where market exists at all points in time, coinciding with the perfectly liquid market tree. The second tree (top, right) reflects the second case, corresponding to vector $\mathbf{y}_{2}$, where market does not exist only at $t=1$. We could have drawn a tree with four branches going directly from the node at $t=0$ to the corresponding four nodes at $t=2$. We prefer the representation above, since we want to make clear that the filtration $\mathcal{F}_{1}$ reflecting the information available at $t=1$ is the same as the filtration $\mathcal{F}_{0}$ reflecting the information available at $t=0$. In a similar way we have a tree representing the vector $\mathbf{y}_{3}$ (low, left) and another one for the vector $\mathbf{y}_{4}$ (low, right). However, if we want to describe all the possible situations in the same tree it will look like the one described below

This super-tree plays a main role in the construction of our superhedging strategies. Actually, our extended filtration will work as if we have an extended tree, coinciding with the one above, where transactions would not be permitted at those nodes represented by open circles. We stress the point 


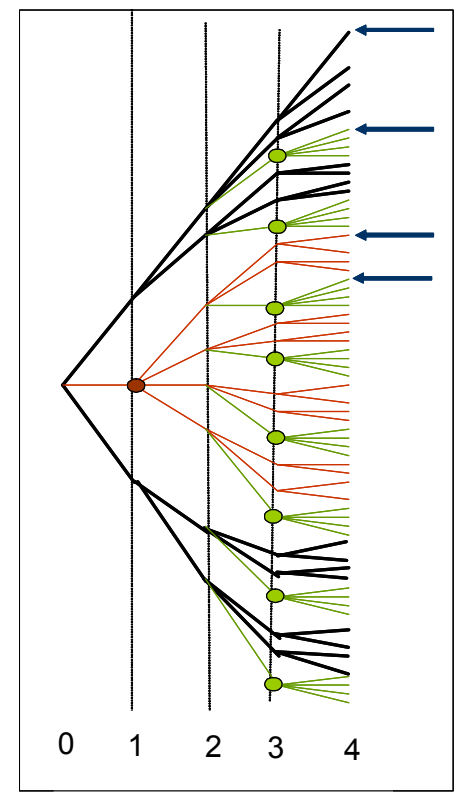

Figure 2: This tree describes all the possibilities under probabilistic illiquidity at $t=1$ and $t=3$. The circles identify the nodes when it is not possible to transact. In the final nodes identified with arrows the stock price is the same. 
that nodes in this tree do not represent mere price realizations. They are rather joint representations of the price process and the market existence process. For instance, the terminal nodes indicated with arrows in the figure are assumed to represent the same price level for the underlying asset, but with different market existence realizations.

We now focus on the construction of the superreplicating strategies in the case of probabilistic illiquidity. At any point in time, the number of shares and the amount invested in the risk-free asset will depend on the existence, or inexistence, of the market at the previous moments in time. However, these values will not depend on the future existence of the market.

Let $\Delta(j, t ; \mathbf{y})$ and $B(j, t ; \mathbf{y})$ denote, respectively, the number of shares and the amount invested in the risk free asset at node $(j, t)$ for a given realization, $\mathbf{y}$, of the process $\left\{y_{s}\right\}_{s \in \mathcal{T}}$. We assume that, if $y_{t}=0$ and $(j, t)$ is an arbitrary successor of $(i, t-1)$, then $\Delta(j, t ; \mathbf{y})=\Delta(i, t-1 ; \mathbf{y})$ and $B(j, t ; \mathbf{y})=B(i, t-1 ; \mathbf{y})$, since the portfolio can not be rebalanced at time $t$. For any given two different sets $\mathbf{y}^{1}$ and $\mathbf{y}^{2}$ with common values $y_{1}^{1}=$ $y_{1}^{2}, y_{2}^{1}=y_{2}^{2}, y_{3}^{1}=y_{3}^{2}, \ldots$ up to time $t$, we assume that

$$
\Delta\left(j, t ; \mathbf{y}^{1}\right)=\Delta\left(j, t ; \mathbf{y}^{2}\right) \text { and } B\left(j, t ; \mathbf{y}^{1}\right)=B\left(j, t ; \mathbf{y}^{2}\right) .
$$

Just as in the deterministic case, let $V(j, t ; \mathbf{y})$ denote the value process generated by such portfolio $[\Delta(j, t ; \mathbf{y}), B(j, t ; \mathbf{y})]$, i.e.,

$$
V(j, t ; \mathbf{y})=\Delta(j, t ; \mathbf{y}) S(j, t)+B(j, t ; \mathbf{y})
$$

Hence,

$$
V\left(j, s ; \mathbf{y}^{1}\right)=V\left(j, s ; \mathbf{y}^{2}\right) .
$$

In an analogous way to the case of probabilistic illiquidity the definition of self-financed strategy and superreplicating strategy is dependent on whether one is in a short or in a long position in the derivative.

In what follows we are going to characterize the upper and lower arbitragefree bounds for the value of an American derivative in the case of probabilistic illiquidity.

\subsection{Some Probabilistic Definitions}

Analogously to what we did in section 2.1, we present some mathematical tools to obtain the arbitrage-free bounds of the American derivative.

We begin by defining $\mathcal{T}_{\mathbf{y}}$ as the subset of points in $\mathcal{T}$ after the last nontrading date. Formally we define $\mathcal{T}_{\mathbf{y}}=\{s \in \mathcal{T}: s \geq \Theta(\mathbf{y})\}$ with

$$
\Theta(\mathbf{y})=\left\{\begin{array}{l}
0 \text { if } y_{t}=1, \forall t \in \mathcal{T} \\
\max \left(m+1: y_{m}=0\right) \text { otherwise. }
\end{array}\right.
$$


Notice that for liquid markets $\mathcal{T}_{\mathbf{y}}=\mathcal{T}$.

Definition 4.1 A node probability measure is a nonnegative function $q(i, t ; \mathbf{y})$, defined for any $\mathbf{y}$ and $(i, t)$ with $t \in \mathcal{T}_{y}$ satisfying

$$
\sum_{(j, t)} \sum_{t \in \mathcal{T}_{y}} \sum_{\mathbf{y}} q(j, t ; \mathbf{y})=1
$$

We now define a specific type of node probability measures.

Definition 4.2 A node probability measure on the event tree is said to be $\mathbf{y}$-simple if, for each $\mathbf{y}$, any $t$ and $t+k \in \mathcal{T}_{y}$, there are no two nodes in the same path, say $(i, t)$ and $(j, t+k) \in i_{t}^{+}(t+k)$, such that $q(i, t ; \mathbf{y})>0$ and $q(j, t+k ; \dot{\mathbf{y}})>0$ where $\dot{\mathbf{y}}$ is any set such that $y_{1}=\dot{y}_{1}, \ldots, y_{t}=\dot{y}_{t}$.

The following theorem is analogous to theorem 2.1 but now in the framework of probabilistic illiquidity. Let $Q(\mathbf{y})$ denote the set of all node probability measures $q(i, t ; \mathbf{y})$.

Theorem 4.1 (Jha and Chalasani) The extreme points of the set of nodes $Q(\mathbf{y})$ are simple node probability measures, i.e., on every path on the event tree there is at most one node where $q$ is strictly positive.

The proof of this theorem follows closely the proof of theorem 2.1 and is presented in Appendix B.

Definition 4.3 An adjusted probability measure is a nonnegative function $p(i, t ; \mathbf{y})$ defined for any $\mathbf{y}$ and $(i, t)$ with $t \in \mathcal{T}_{y}$ such that $p(0,0)=$ $p(0,0 ; \mathbf{y})=1$ and

$$
p(i, t ; \mathbf{y})=\sum_{(j, s) \in i_{t}^{+}(s)} \sum_{\left\{\mathbf{z}: z_{0}=y_{0}, \ldots, z_{t}=y_{t}\right\}} p(j, s ; \mathbf{z}),
$$

with $\mathbf{s}=\min \left\{n \in \mathcal{T}_{\mathbf{z}}: y_{n}=1\right.$ and $\left.n>t\right\}$.

Let the set of all probability measures be denoted by $\mathbb{P}_{y}$. Also, let $\tau_{y}$ denote an ordinary stopping time that is conditional on the realization of the process $\left\{y_{t}\right\}_{t \in \mathcal{T}}$. For any $\mathbf{y}, \tau_{y}$ is a map that is defined from $\Omega$ to $\left\{s \in \mathcal{T}: y_{s}=1\right\}$ such that $\{w: \tau(w ; \mathbf{y}) \leq t\} \in \mathcal{F}_{t}$ for all $t \in\left\{s \in \mathcal{T}: y_{s}=1\right\}$. Additionally, for two different sets $\mathbf{y}^{1}$ and $\mathbf{y}^{2}$ with common values $y_{1}^{1}=$ $y_{1}^{2}, y_{2}^{1}=y_{2}^{2}, \ldots$, up to time $t$, if $\tau\left(w ; \mathbf{y}^{1}\right)=t$ then $\tau\left(w ; \mathbf{y}^{2}\right)=t$. We define a nonnegative adapted process $X_{\tau, y}$ associated with the stopping time that has the form $X_{\tau}[i, k ; \mathbf{y}]=1$ if $\tau(w ; \mathbf{y})=k$ and $X_{\tau}[i, k ; \mathbf{y}]=0$ otherwise. Let $\mathbb{T}_{y}$ and $\mathbb{X}_{\mathbb{T}, y}$ denote the set of all $\tau(\mathbf{y})$ and associated $X_{\tau}(\mathbf{y})$, respectively. 
Definition 4.4 A $\mathbf{y}$-simple node probability measure is said to be associated with a given stopping time if $q(i, t ; \mathbf{y})$ is equal to zero when $X_{\tau}(i, t ; \mathbf{y})$ is equal to zero, and $q(i, t ; \mathbf{y})$ is positive when $X_{\tau}(i, k ; \mathbf{y})$ is strictly positive, for any $\mathbf{y}$ and node $(i, t)$.

Let the set of all node probability measures with this property be denoted by $Q^{\tau}(\mathbf{y})$.

Definition 4.5 For any probability measure $P_{y} \in \mathbb{P}_{y}$ and stopping time $\tau_{y} \in$ $\mathbb{T}_{y}$ we say that $P_{y}$ is a $\tau_{y}$-martingale measure if, $P_{y}$-almost surely, for any $(i, t)$ and $\mathbf{y}$ such that $y_{t}=1$ we have

$$
\sum_{(j, s) \in i^{+}(\mathbf{s})} \sum_{s>t, s \in \mathcal{T}_{z}} \sum_{\left\{\mathbf{z}: z_{0}=y_{0}, \ldots, z_{t}=y_{t}\right\}} p(j, s ; \mathbf{z}) X_{\tau}(j, s ; \mathbf{z})[\bar{S}(i, t)-\bar{S}(j, s)]=0
$$

The set of all $P_{y}$ that have this property is denoted by $\mathcal{P}_{y}\left(\tau_{y}\right)$

Let $\left(P_{y}, X_{\tau, y}\right)$ denote a measure-strategy pair, i.e., a pair constituted by an adjusted probability measure and a nonnegative adapted process.

Definition 4.6 A measure-strategy pair $\left(P_{y}, X_{\tau, y}\right)$ is said to be equivalent to a node probability measure if, for any given node $(i, t)$ with $t \in \mathcal{T}_{y}$, $p(i, t ; \mathbf{y}) X_{\tau}(i, t ; \mathbf{y})=q(i, t ; \mathbf{y})$.

We can now enunciate the following result, adapted from Jha and Chalasani (2001) to include the random variable $y$.

Theorem 4.2 Consider a node probability measure $q \in Q(\mathbf{y})$. Then there exists a measure-strategy pair $\left(P_{y}, \tau_{y}\right)$ equivalent to $q$, where for any given $\mathbf{y}$, $P_{y}$ is uniquely defined at node $(i, t)$ where

$$
q(i, t ; \mathbf{y})+\sum_{(j, s) \in i^{+}(\mathbf{s})} \sum_{s>t, s \in \mathcal{T}_{z}} \sum_{\left\{\mathbf{z}: z_{0}=y_{0}, \ldots, z_{t}=y_{t}\right\}} q(j, s ; \mathbf{z})
$$

is strictly positive and, for a given $\mathbf{y}, \tau_{\mathbf{y}}$ is uniquely defined at nodes $(i, t)$ where $q(i, t ; \mathbf{y})$ is defined and is strictly positive. Conversely, if $\left(P_{y}, \tau_{y}\right)$ is a measure strategy-pair, then the node function $q \in Q(\mathbf{y})$ such that $q(i, t ; \mathbf{y})=$ $p(i, t ; \mathbf{y}) X(i, t ; \mathbf{y})$ is the unique equivalent node-measure.

Proof. The proof of this theorem is a modification of the one provided in Jha and Chalasani (2001). See appendix.

All the mathematical definitions provided above are dependent of $y$. In what follows we will shown that it is possible to define an adjusted probability and a randomized stopping time in the original tree that is closely related with the concepts just presented. 
Definition 4.7 An adjusted probability measure $\overline{\mathbf{P}}(i, t)$ is a nonnegative function such that $\bar{P}(0,0)=1$ and $\bar{P}(i, t)=\sum_{(j, t+1) \in i_{t}^{+}} \bar{P}(j, t+1)$, for all $t \in \mathcal{T}$.

The set of all probability measures $\bar{P}$ is denoted by $\overline{\mathbb{P}}$.

A randomized stopping time is a nonnegative adapted process $X$ with the property that on every path of the event tree the sum of the random variable is equal to one, i.e.,

$$
\sum_{t \in \mathcal{T}} X\left(i_{t}, t\right)=1
$$

where $i_{t+1} \in i_{t}^{+}$. The set of all randomized stopping time is denoted by $\mathbb{X}$.

Definition 4.8 An adjusted probability measure $\bar{P} \in \overline{\mathbb{P}}$ is said to be a $X_{y^{-}}$ martingale measure if there is a randomized stopping time $X \in \mathbb{X}$, a stopping time $\tau_{y} \in \mathbb{T}_{y}$ and an adjusted probability measure $P_{y} \in \mathcal{P}_{y}\left(\tau_{y}\right)$ such that

$$
X(i, t) \bar{P}(i, t)=\sum_{\left\{\mathbf{y}: y_{t}=1\right\}} p(i, t ; \mathbf{y}) X_{\tau}(i, t ; \mathbf{y})
$$

for any $(i, t)$ with $t \in \mathcal{T}$.

The set of all $\bar{P}$ that are $X_{y}$ martingale measures is denoted by $\overline{\mathcal{P}}\left(X_{y}\right)$.

Theorem 4.3 For any given $\tau_{y}$-martingale measure, $P_{y} \in \mathcal{P}_{y}\left(\tau_{y}\right)$, the adjusted probability measure $\bar{P} \in \overline{\mathbb{P}}$ and the randomized stopping time $X \in \mathbb{X}$ such that $\bar{P}$ is a $X_{y}$ martingale measure are as follow. The adjusted probability measure is such that $\bar{P}(0,0)=1$ and for any $(i, t) \in j_{t-1}^{+}(t)$ such that $\alpha(j, t-1) \neq 0$, where

$$
\begin{aligned}
\alpha(i, t)= & \sum_{r \geq t} \sum_{\left\{\mathbf{z}: z_{t}=1\right\}} p(i, r ; \mathbf{z}) X_{\tau}(i, r ; \mathbf{z})+ \\
& \sum_{r \geq s} \sum_{(j, s) \in i_{t-1}^{+}} \sum_{\left\{\mathbf{z}: z_{t} \neq 1\right\}} p(j, r ; \mathbf{z}) X_{\tau}(j, r ; \mathbf{z})
\end{aligned}
$$

and $s=\min \left\{r \in \mathcal{T}: r>t\right.$ and $\left.X_{\tau}(j, r ; \mathbf{z})=1\right\}$ then,

$$
\bar{P}(i, t)=\bar{P}(j, t-1) \frac{\alpha(i, t)}{\sum_{(i, t) \in j_{t-1}^{+}(t)} \alpha(i, t)} .
$$

If $\sum_{(i, t) \in j_{t-1}^{+}(t)} \alpha(i, t)=0$ then $\bar{P}(i, t)=\bar{P}(j, t-1)$ for a given successor $(i, t)$ of $(j, t-1)$ and zero for all others successors of $(j, t-1)$. 
The randomized stopping time $X \in \mathbb{X}$ is uniquely defined for any node $(i, t)$ such that $\bar{P}(i, t) \neq 0$ and is given by

$$
X(i, t)=\frac{\sum_{\left\{\mathbf{y}: y_{t}=1\right\}} p(i, t ; \mathbf{y}) X_{\tau}(i, t ; \mathbf{y})}{\bar{P}(i, t)} .
$$

If $\bar{P}(i, t)=0$ but there is a predecessor $(k, t-1)$ such that $\bar{P}(k, t-1) \neq 0$ take

$$
X(i, t)=\frac{\sum_{(i, t) \in k_{t-1}^{+}(t)} \alpha(i, t)}{\bar{P}(k, t-1)} .
$$

Otherwise, $X(i, t)=0$.

Proof. See Appendix B3.

\section{Results on Probabilistic Illiquidity}

\subsection{Upper bound for the Value of an American Deriva- tive}

The upper bound for the value of an American derivative is the maximum value for which the derivative would be transacted without allowing for arbitrage opportunities. As described in the deterministic illiquidity case, in order to find the upper bound consider a short position in the derivative. The maximum value for which the derivative would be transacted without allowing for arbitrage opportunities would be the value of the cheapest selffinanced portfolio that the buyer of the derivative can buy in order to completely hedge against any possibility of exercise of the American derivative.

A strategy is said to be a self-financed strategy if for any given $\mathbf{y}$ the portfolio at node $\left(j, t_{1}\right)$, where $t_{1} \in\left\{t \in \mathcal{T}: y_{t}=1\right\}$, generates in $t_{2}$ a value $\Delta\left(j, t_{1} ; \mathbf{y}\right) S\left(i, t_{2}\right)+B\left(j, t_{1} ; \mathbf{y}\right) R^{t_{2}-t_{1}}$ such that

$$
\Delta\left(j, t_{1} ; \mathbf{y}\right) S\left(i, t_{2}\right)+B\left(j, t_{1} ; \mathbf{y}\right) R^{t_{2}-t_{1}} \geq V\left(i, t_{2} ; \mathbf{y}\right)
$$

with $\left(i, t_{2}\right) \in j_{t_{1}}^{+}\left(t_{2}\right)$ and $t_{2}=\min \left\{s \in \mathcal{T}: s>t\right.$ and $\left.y_{s}=1\right\}$.

A sequence of portfolios $\{[\Delta(j, t ; \mathbf{y}), B(j, t ; \mathbf{y})]\}_{t \in \mathcal{T}}$, one for each $\mathbf{y}$, is said to be a superreplicating strategy if its value is higher than or equal to the payoff of the derivative at any node in the next transaction time. In other words, for any trading dates $t_{1}$ and $t_{2}$ such that $t_{1} \in\left\{t \in \mathcal{T}: y_{t}=1\right\}$ and $t_{2}=\min \left\{t \in \mathcal{T}: t>t_{1}\right.$ and $\left.y_{t}=1\right\}$ and arbitrary nodes, $\left(j, t_{1}\right)$ and $\left(i, t_{2}\right) \in$ 
$j_{t_{1}}^{+}\left(t_{2}\right)$, the portfolio at $t_{1},\left[\Delta\left(j, t_{1} ; \mathbf{y}\right), B\left(j, t_{1} ; \mathbf{y}\right)\right]$, must be such as to generate in $t_{2}$ a value $\Delta\left(j, t_{1} ; \mathbf{y}\right) S\left(i, t_{2}\right)+B\left(j, t_{1} ; \mathbf{y}\right) R^{t_{2}-t_{1}}$ such that

$$
\Delta\left(j, t_{1} ; \mathbf{y}\right) S\left(i, t_{2}\right)+B\left(j, t_{1} ; \mathbf{y}\right) R^{t_{2}-t_{1}} \geq G\left(i, t_{2}\right) .
$$

Since it is the cheapest initial portfolio, the upper bound $V_{p}^{u}$ must satisfy

$$
V_{p}^{u}=\min V(0,0) \text {. }
$$

The decision variables are the $\Delta(j, t ; \mathbf{y})$ and $B(j, t ; \mathbf{y})$ for all non-terminal nodes of the event tree. However, this optimization is subject to the constraints of self-financing (18) and superreplication (19).

More formally, for any given $\mathbf{y}$ take any $t_{1} \in \mathcal{T}$ such that $y_{t_{1}}=1$. Define the consecutive trading date $t_{2}$ such that $t_{2}=\min \left(s \in \mathcal{T}: s>t_{1}\right.$ and $\left.y_{s}=1\right)$. The upper bound for the value of the American derivative can thus be seen as the solution of the following problem:

$$
V_{p}^{u}=\min _{\{\Delta(j, t ; \mathbf{y}), B(j, t ; \mathbf{y})\}_{t \in\left\{s \in \mathcal{T}: y_{s}=1\right\} \backslash\{T\}}} \Delta(0,0) S(0,0)+B(0,0)
$$

subject to the superreplicating restrictions:

$$
\begin{gathered}
\Delta(0,0) S(0,0)+B(0,0) \geq G(0,0) \\
\Delta\left(j, t_{1} ; \mathbf{y}\right) S\left(i, t_{2}\right)+B\left(j, t_{1} ; \mathbf{y}\right) R^{t_{2}-t_{1}} \geq G\left(i, t_{2}\right),
\end{gathered}
$$

and subject to the self-financing restrictions:

$$
\Delta\left(j, t_{1} ; \mathbf{y}\right) S\left(i, t_{2}\right)+B\left(j, t_{1} ; \mathbf{y}\right) R^{t_{2}-t_{1}} \geq \Delta\left(i, t_{2} ; \mathbf{y}\right) S\left(i, t_{2}\right)+B\left(i, t_{2} ; \mathbf{y}\right)
$$

for any $\left(i, t_{2}\right) \in j_{t_{1}}^{+}\left(t_{2}\right)$.

Using results from linear programming the upper bound arbitrage free bound of the American derivative can be written as follows.

Theorem 5.1 There is a node probability measure $q \in Q(y)$ such that the upper hedging price of an American derivative in a probabilistic dry market can be written as

$$
V_{p}^{u}=\max _{q \in Q(y)} \sum_{(j, t)} \sum_{t \in \mathcal{T}_{y}} \sum_{\mathbf{y}} q(j, t ; \mathbf{y}) \bar{G}(j, t)
$$

with

$$
\sum_{(j, s) \in i_{t}^{+}(\mathbf{s})} \sum_{s>t, s \in \mathcal{T}_{z}} \sum_{\left\{\mathbf{z}: z_{0}=y_{0}, \ldots, z_{t}=y_{t}\right\}} p(j, s ; \mathbf{z}) X_{\tau}(j, s ; \mathbf{z})[\bar{S}(i, t)-\bar{S}(j, s)]=0
$$


Proof. This proof follows the methodology used in theorem (3.2). As the upper bound for the value of the American derivative, $V_{p}^{u}$, is the solution of linear programming problem it is possible to construct its dual. Let $\lambda(0,0)$, $\lambda\left(i, t_{2} ; \mathbf{y}\right)$ and $\gamma\left(i, t_{2} ; \mathbf{y}\right)$ denote the dual variables that are associated, respectively, with the restrictions (20), (21) and (22) of the primal problem. Note that, as we assume that given two different sets $\mathbf{y}^{1}$ and $\mathbf{y}^{2}$ with common values $y_{1}^{1}=y_{1}^{2}, y_{2}^{1}=y_{2}^{2}, y_{3}^{1}=y_{3}^{2}, \ldots$ up to time $t_{1}$, the portfolio will be the same, i.e.,

$$
\Delta\left(j, t_{1} ; \mathbf{y}^{1}\right)=\Delta\left(j, t_{1} ; \mathbf{y}^{2}\right) \text { and } B\left(j, t_{1} ; \mathbf{y}^{1}\right)=B\left(j, t_{1} ; \mathbf{y}^{2}\right),
$$

then, $\lambda\left(i, t_{2} ; \mathbf{y}^{1}\right)=\lambda\left(i, t_{2} ; \mathbf{y}^{2}\right)$ for all $\left(i, t_{2}\right) \in i_{t_{1}}^{+}\left(t_{2}\right)$. Before presenting the dual problem let define

$$
\Theta_{t}=\left\{\mathbf{y}: y_{t}=1 \text { and } \min \left[s \in \mathcal{T}_{y}: s>t\right]=\min \left[s \in \mathcal{T}: y_{s}=1 \text { and } s>t\right]\right\}
$$

The dual problem is given by

$$
\max _{q \in Q(y)} \sum_{(j, t)} \sum_{t \in \mathcal{T}_{y}} \sum_{\mathbf{y}} \lambda(j, t ; \mathbf{y}) \bar{G}(j, t)
$$

subject to the conditions:

$\lambda(0,0) S(0,0)+\sum_{(j, s) \in i_{0}^{+}(t)} \sum_{z \in \Theta_{0}}[\lambda(j, t ; \mathbf{z})+\gamma(j, t ; \mathbf{z})] S(j, t)=S(0,0)$,

$$
\lambda(0,0)+\sum_{(j, s) \in i_{0}^{+}(t)} \sum_{\mathbf{z} \in \Theta_{0}}[\lambda(j, t ; \mathbf{z})+\gamma(j, t ; \mathbf{z})] R^{t}=1
$$

where $t=\min \left\{s \in \mathcal{T}_{z}: s>0\right\}$.

For any $(i, t)$ and $\mathbf{y}$ such that $t \in \mathcal{T}_{y} \backslash\left\{0, \max \left[r \in \mathcal{T}_{y}\right.\right.$ and $\left.\left.r<T\right], T\right\}$,

$$
\sum_{(j, s) \in i_{t}^{+}(s)} \sum_{z \in \Theta_{t}}[\lambda(j, s ; \mathbf{z})+\gamma(j, s ; \mathbf{z})] S(j, s)=\gamma(i, t ; \mathbf{y}) S(i, t)
$$

and

$$
\sum_{(j, s) \in i_{t}^{+}(s)} \sum_{z \in \Theta_{t}}[\lambda(j, s ; \mathbf{z})+\gamma(j, s ; \mathbf{z})] R^{s-t}=\gamma(i, t ; \mathbf{y})
$$

where $s=\min \left\{r \in \mathcal{T}_{z}: r>t\right\}$. Finally, for any $(i, t)$ and $\mathbf{y}$ such that $t=$ $\max \left\{r \in \mathcal{T}_{y}\right.$ and $\left.r<T\right\}$

$$
\sum_{(j, T) \in i_{t}^{+}(T)} \sum_{z \in \Theta_{t}} \lambda(j, T ; \mathbf{z}) S(j, T)-\gamma(i, t ; \mathbf{y}) S(i, t)=0
$$


and

$$
\sum_{(j, T) \in i_{t}^{+}(T)} \sum_{z \in \Theta_{t}} \lambda(j, T ; \mathbf{z}) R^{T-t}-\gamma(i, t ; \mathbf{y})=0
$$

The restrictions presented in equations (25) and (27) can be rewritten as

$$
S(i, t) \gamma(i, t)=\sum_{r \geq s} \sum_{(j, r) \in i_{t}^{+}(r)} \sum_{z \in \Theta_{t}} \lambda(j, r ; \mathbf{z}) S(j, r)
$$

and the restrictions presented in equations (26) and (28) can be rewritten as

$$
\gamma(i, t)=\sum_{r \geq s} \sum_{(j, s) \in i_{t}^{+}(s)} \sum_{z \in \Theta_{t}} \lambda(j, r ; \mathbf{z}) R^{r-t} .
$$

for all $(i, t), t \in \mathcal{T} \backslash\{0, T\}$ and with $s=\min \left\{r \in \mathcal{T}_{z}: r>t\right\}$. The two previous equations can be written as

$$
\begin{aligned}
& S(i, t) \sum_{r \geq s} \sum_{(j, s) \in i_{t}^{+}(s)} \sum_{z \in \Theta_{t}} \lambda(j, r ; \mathbf{z}) R^{r-t} \\
= & \sum_{r \geq s} \sum_{(j, s) \in i_{t}^{+}(s)} \sum_{z \in \Theta_{t}} \lambda(j, r ; \mathbf{z}) S(j, r)
\end{aligned}
$$

Taking into account equations (23) and (24) we obtain, for all $t \in \mathcal{T} \backslash\{T\}$

$$
\begin{aligned}
& S(i, t) \sum_{r \geq s} \sum_{(j, s) \in i_{t}^{+}(s)} \sum_{z \in \Theta_{t}} \lambda(j, r ; \mathbf{z}) R^{r-t} \\
= & \sum_{r \geq s} \sum_{(j, s) \in i_{t}^{+}(s)} \sum_{z \in \Theta_{t}} \lambda(j, r ; \mathbf{z}) S(j, r)
\end{aligned}
$$

with

$$
\sum_{t \in \mathcal{T}_{y}} \sum_{(j, t)} \sum_{\mathbf{y}} \lambda(j, t ; \mathbf{y}) R^{t}=1
$$

Let $q(i, t ; \mathbf{y})=\lambda(i, t ; \mathbf{y}) R^{t}$.then,

$$
\begin{aligned}
& \bar{S}(i, t) \sum_{r \geq s} \sum_{(j, s) \in i_{t}^{+}(s)} \sum_{z \in \Theta_{t}} \lambda(j, r ; \mathbf{z}) R^{r-t} \\
= & \sum_{r \geq s} \sum_{(j, s) \in i_{t}^{+}(s)} \sum_{z \in \Theta_{t}} \lambda(j, r ; \mathbf{z}) \bar{S}(j, r)
\end{aligned}
$$

and

$$
\sum_{t \in \mathcal{T}_{y}} \sum_{(j, t)} \sum_{\mathbf{y}} q(j, t ; \mathbf{y})=1
$$

The upper bound solving the problem above can also be seen as the solution of a more intuitive problem. In fact, it can be shown that this upper bound maximizes over all possible stopping times the expected discounted payoff, when the expectation is optimized among all adjusted probability measures. In other words, 
Theorem 5.2 There is an adjusted probability measure $P_{y} \in \mathcal{P}_{y}\left(\tau_{y}\right)$ and an adapted process $X_{\tau, y} \in \mathbb{X}_{\mathbb{T}, y}$ such that the upper hedging price of an American derivative in a probabilistic dry market can be written as

$$
V_{p}^{u}=\max _{X_{\tau, y} \in \mathbb{X}_{\mathbb{T}, y}} \max _{P_{y} \in \mathcal{P}_{y}\left(\tau_{y}\right)} E^{\bar{p}} G_{X_{\tau, y}}
$$

where $G_{X_{\tau, y}}(i, t)=G(i, t) X_{\tau, y}(i, t)$

Proof. In an analogous way to the proof of theorem (3.2), using theorem (4.1) and theorem (4.2) the conclusion is straightforward.

Note that this result is the same that would be obtained if the filtration that describes the stock price is an augmented one, in the spirit of the one presented in figure (2), with no uncertainty about the existence of the market and no transactions in some nodes (the ones identified in the figure).

However, the upper bound of the value of an American derivative can also be written using randomized stopping times if an adjusted probability measure with an additional characteristic is considered. The adjusted probability measure have to be decomposed in such a way that if an augmented filtration is considered the stock price is a martingale.

If the initial filtration is considered it is not possible to write the upper bound as an optimization over ordinary stopping times, as in theorem (5.2). In this case, randomized stopping times may be needed.

Theorem 5.3 There is an adjusted probability measure $\bar{P} \in \overline{\mathcal{P}}\left(X_{y}\right)$ and a process $X \in \mathbb{X}$ such that the upper hedging price of an American derivative in a probabilistic dry market can be written as

$$
V_{p}^{u}=\max _{X \in \mathbb{X}} \max _{\bar{P} \in \overline{\mathcal{P}}\left(X_{y}\right)} E^{\bar{p}} G_{X}
$$

with $G_{X}(i, t)=G(i, t) X(i, t)$.

Proof. This result follows from the application of theorem (4.3) to the result presented in theorem (5.2).

In what follows we are going to consider an example. The upper bound of the American derivative is obtained using the primal and the dual problem. In this example no optimal pure stopping time exists that maximizes the expected value of the payoffs of the American derivative. The expected value of the payoffs of the American derivative is maximized with randomized stopping times.

Consider $T=\{0,1,2\}, \mathcal{T}=\{0,2\}$ and $\mathcal{T}_{p}=\{1\}$. Let $R=1$ and the uncertainty about the price of the underlying stock and the derivative be given by 


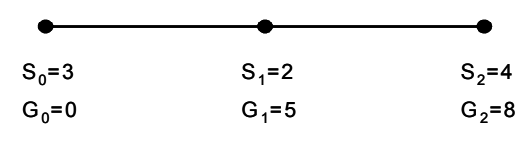

Figure 3:

There are two sets $\mathbf{y}, \mathbf{y}_{1}=\{1,1,1\}$ and $\mathbf{y}_{2}=\{1,0,1\}$. The optimum value of the variables in the primal problem is

$$
\begin{aligned}
& \Delta\left(0,0 ; \mathbf{y}_{1}\right)=\Delta\left(0,0 ; \mathbf{y}_{2}\right)=\Delta(0,0)=1,5 \\
& B\left(0,0 ; \mathbf{y}_{1}\right)=B\left(0,0 ; \mathbf{y}_{2}\right)=B(0,0)=2 \\
& \Delta\left(0,1 ; \mathbf{y}_{1}\right)=2,5 \\
& B\left(0,1 ; \mathbf{y}_{1}\right)=0
\end{aligned}
$$

that results in an optimum value of the function

$$
\Delta(0,0) S(0,0)+B(0,0)=6,5 .
$$

In what concerns the dual problem the optimum value of the variables

$$
\begin{aligned}
& q(0,0)=q\left(0,0 ; \mathbf{y}_{1}\right)=q\left(0,0 ; \mathbf{y}_{2}\right)=0 \\
& q(0,1)=q\left(0,1 ; \mathbf{y}_{1}\right)=0.5 \\
& q(0,2)=q\left(0,2 ; \mathbf{y}_{1}\right)+q\left(0,2 ; \mathbf{y}_{2}\right)=0+0.5=0.5
\end{aligned}
$$

As a result, the optimum value of the objective function is

$$
q(0,1) G(0,1)+q(0,2) G(0,2)=6.5 .
$$

In this case the probability measure $P_{y}$ is given by

$$
\begin{aligned}
& p_{y}(0,0)=1 \\
& p_{y}\left(0,1, \mathbf{y}_{1}\right)=1 * \frac{0.5}{0.5+0.5}=0.5 \\
& p_{y}\left(0,2, \mathbf{y}_{1}\right)=0.5 \\
& p_{y}\left(0,2, \mathbf{y}_{2}\right)=0.5
\end{aligned}
$$

and the stopping time $\tau_{y}$ is such that $X_{\tau}$ is given by

$$
\begin{aligned}
& X_{\tau}(0,0)=0 \\
& X_{\tau}\left(0,1, \mathbf{y}_{1}\right)=1 \\
& X_{\tau}\left(0,1, \mathbf{y}_{2}\right)=0 \\
& X_{\tau}\left(0,2, \mathbf{y}_{1}\right)=0 \\
& X_{\tau}\left(0,2, \mathbf{y}_{2}\right)=1
\end{aligned}
$$


The probability measure $\bar{P}$ is

$$
P(0,0)=P(0,1)=P(0,2)=1
$$

and the randomized stopping time $X$

$$
\begin{aligned}
& X(0,0)=0 \\
& X(0,1)=0.5 \\
& X(0,2)=0.5
\end{aligned}
$$

The use of the randomized stopping times is closed related to the fact that we are using the filtration that reflects the information that is available if the market is completely liquid.

\subsection{Lower bound for the Value of an American Deriva- tive}

The lower bound for the value of an American derivative is the minimum value for which the derivative would be transacted without allowing for arbitrage opportunities. As in the deterministic illiquidity case, in order to find the lower bound consider a long position in the derivative. For a given exercise policy consider the most expensive self-financed portfolio that the buyer of the American derivative can buy in order to be fully hedged. The minimum value for which the derivative would be transacted without allowing for arbitrage opportunities would be the value of most expensive portfolio chosen among all the portfolios just mentioned.

For any given exercise policy $\tau_{y}$ and any node $(j, t)$, such that $(j, t)$ is before the exercise time, consider the portfolio constituted of $\Delta^{\tau_{y}}(j, t ; \mathbf{y})$ shares of the underlying asset and an amount $B^{\tau_{y}}(j, t ; \mathbf{y})$ invested in the risk free asset. Its value process is given by

$$
V^{\tau_{y}}\left(i, t_{2} ; \mathbf{y}\right)=\Delta^{\tau_{y}}\left(i, t_{2} ; \mathbf{y}\right) S\left(i, t_{2}\right)+B^{\tau_{y}}\left(i, t_{2} ; \mathbf{y}\right)
$$

For a long position in the derivative, a strategy is said to be a self-financed strategy if for an any given $\mathbf{y}$ the portfolio at node $\left(j, t_{1}\right)$, where $t_{\mathbf{1}} \in$ $\left\{t \in \mathcal{T}: y_{t}=1\right\}$, generates in $t_{2}$ a value such that

$$
\Delta^{\tau_{y}}\left(j, t_{1} ; \mathbf{y}\right) S\left(i, t_{2}\right)+B^{\tau_{y}}\left(j, t_{1} ; \mathbf{y}\right) R^{t_{2}-t_{1}} \leq V^{\tau_{y}}\left(i, t_{2} ; \mathbf{y}\right),
$$

with $\left(i, t_{2}\right) \in j_{t_{1}}^{+}\left(t_{2}\right), t_{2}=\min \left\{s \in \mathcal{T}: s>t\right.$ and $\left.y_{s}=1\right\}$ such that there is a node $\left(m, t_{3}\right) \in j_{t_{2}}^{+}\left(t_{3}\right)$ with $t_{3}$ such $X_{\tau}\left(m, t_{3}\right)=1$. 
A sequence of portfolios $\left\{\left[\Delta^{\tau_{y}}(j, t ; \mathbf{y}), B^{\tau_{y}}(j, t ; \mathbf{y})\right]\right\}_{t \in \mathcal{T}}$, one for each $\mathbf{y}$, is said to be a superreplicating strategy if its value is higher than or equal to the payoff of the derivative at any node in the next transaction time. In other words, for any trading dates $t_{1}$ and $t_{2}$ such that $t_{1} \in\left\{t \in \mathcal{T}: y_{t}=1\right\}$ and $t_{2}=\min \left\{t \in \mathcal{T}: t>t_{1}\right.$ and $\left.y_{t}=1\right\}$ and arbitrary nodes, $\left(j, t_{1}\right)$ and $\left(i, t_{2}\right) \in$ $j_{t_{1}}^{+}\left(t_{2}\right)$ such that $X_{\tau}\left(i, t_{2}\right)=1$, the portfolio at $t_{1},\left[\Delta^{\tau}\left(j, t_{1} ; \mathbf{y}\right), B^{\tau}\left(j, t_{1} ; \mathbf{y}\right)\right]$, must be such as to generate in $t_{2}$ a value such that

$$
\Delta^{\tau_{y}}\left(j, t_{1} ; \mathbf{y}\right) S\left(i, t_{2}\right)+B^{\tau_{y}}\left(j, t_{1} ; \mathbf{y}\right) R^{t_{2}-t_{1}} \leq G_{\tau_{y}}\left(i, t_{2}\right) .
$$

As described above, this would be the value of the most expensive selffinancing, "superreplicating" portfolio. Since it is the most expensive initial portfolio, the upper bound $V^{u}$ must satisfy

$$
V_{p}^{l}=\max _{\tau_{y}} V^{\tau_{y}}(0,0)
$$

The decision variables are the $\Delta^{\tau}(j, t ; \mathbf{y})$ and $B^{\tau}(j, t ; \mathbf{y})$ for all non-terminal nodes of the event tree. However, this optimization is subject to the constraints of self-financing (29) and superreplication (30).

More formally, for any given $\mathbf{y}$ take any $t_{1} \in \mathcal{T}$ such that $y_{t_{1}}=1$. Define the consecutive trading date as $t_{2}=\min \left(s \in \mathcal{T}: s>t_{1}\right.$ and $\left.y_{s}=1\right)$. The lower bound for the value of the American derivative can thus be seen as the solution of the following problem:

$$
V_{p}^{l}=\max _{\tau_{y} \in \mathbb{T}_{y}} \max _{\{\Delta(j, t ; \mathbf{y}), B(j, t ; \mathbf{y})\}_{t \in\left\{s \in \mathcal{T}: y_{s}=1\right\} \backslash\{T\}}} \Delta^{\tau_{y}}(0,0) S(0,0)+B^{\tau_{y}}(0,0)
$$

subject to the superreplicating restriction

$$
\Delta^{\tau_{y}}(0,0) S(0,0)+B^{\tau_{y}}(0,0) \leq G_{\tau}(0,0),
$$

if $X_{\tau_{y}}(0,0)=1$. However, if $X_{\tau_{y}}(0,0)=0$, the superreplication condition is defined for any node $\left(i, t_{2}\right)$ such that $X\left(i, t_{2}\right)=1$, and is given by

$$
\Delta^{\tau_{y}}\left(j, t_{1}\right) S\left(i, t_{2}\right)+B^{\tau_{y}}\left(j, t_{1}\right) R^{t_{2}-t_{1}} \leq G_{\tau_{y}}\left(i, t_{2}\right),
$$

for any $t_{1} \in \mathcal{T}_{m} \backslash\{T\}$ such that $\left(i, t_{2}\right) \in j^{+}\left(t_{2}\right)$ and $t_{2}=\min \left(s \in \mathcal{T}_{m}: s>t_{1}\right)$.

Additionally, for any node $\left(i, t_{2}\right)$ such that $X_{\tau_{y}}\left(i, t_{2}\right)=1$ the self-financing conditions apply, i.e.,

$$
\Delta^{\tau_{y}}\left(k, t_{1}\right) S^{\tau_{y}}\left(i, t_{2}\right)+B^{\tau_{y}}\left(k, t_{1}\right) R^{t_{2}-t_{1}} \leq \Delta^{\tau_{y}}\left(i, t_{2}\right) S\left(i, t_{2}\right)+B^{\tau_{y}}\left(i, t_{2}\right)
$$

for all $t_{1} \in \mathcal{T}_{m} \backslash\left\{\max \left\{t \in \mathcal{T}_{m}: t<T\right\}, T\right\}$ and $\left(i, t_{2}\right) \in j^{+}\left(t_{2}\right)$ with $t_{2}=$ $\min \left(s \in \mathcal{T}_{m} \backslash\{T\}: s>t_{1}\right)$.

Using results from linear programming the upper bound arbitrage free bound of the American derivative can be written as follows. 
Theorem 5.4 There is a node probability measure $q \in Q^{\tau}(y)$ and a process $\tau_{y} \in \mathbb{T}_{y}$ such that the upper hedging price of an American derivative in a probabilistic dry market can be written as

$$
V_{p}^{l}=\max _{\tau_{y} \in \mathbb{T}_{y}} \min _{q \in Q^{\tau}(y)} \sum_{(j, t)} \sum_{t \in \mathcal{T}_{y}} \sum_{\mathbf{y}} q(j, t ; \mathbf{y}) \bar{G}_{\tau_{y}}(j, t)
$$

such that for any $(i, t)$ and $t \in \mathcal{T}$

$$
\sum_{m>t, m \in \mathcal{T}_{m}} \sum_{(j, m) \in i_{t}^{+}(m)} q(j, m)[\bar{S}(i, t)-\bar{S}(j, m)]=0 .
$$

Proof. For a given stopping time the problem that must be solved in order to find the lower bound for the value of the American derivative can be rewritten as

$$
V_{p}^{l}=\min _{q \in Q^{\tau}(y)} \sum_{(j, t)} \sum_{t \in \mathcal{T}_{y}} \sum_{\mathbf{y}} q(j, t ; \mathbf{y}) \bar{G}_{\tau_{y}}(j, t)
$$

such that for any $(i, t)$ and $t \in \mathcal{T}_{m}$

$$
\sum_{m>t, m \in \mathcal{T}_{m}} \sum_{(j, m) \in i_{t}^{+}(m)} q(j, m)[\bar{S}(i, t)-\bar{S}(j, m)]=0 .
$$

Considering the optimization with respect to $\tau_{y}$ the problem becomes

$$
\max _{\tau_{y} \in \mathbb{T}_{y}} \min _{q \in Q^{\tau}} \sum_{(j, t)} \sum_{t \in \mathcal{T}_{y}} \sum_{\mathbf{y}} q(j, t ; \mathbf{y}) \bar{G}_{\tau_{y}}(j, t)
$$

such that for any $(i, t)$ with $t \in \mathcal{T}$

$$
\sum_{m>t, m \in \mathcal{T}_{m}} \sum_{(j, m) \in i_{t}^{+}(m)} q(j, m)[\bar{S}(i, t)-\bar{S}(j, m)]=0 .
$$

Theorem 5.5 There is an adjusted probability measure $\bar{P} \in \overline{\mathcal{P}}\left(X_{y}\right)$ and a process $\tau_{y} \in \mathbb{T}_{y}$ such that the lower hedging price of an American derivative in a probabilistic dry market can be written as

$$
V_{p}^{l}=\max _{\tau_{y} \in \mathbb{T}_{y}} \min _{\bar{P} \in \overline{\mathcal{P}}\left(X_{y}\right)} E^{p} G_{\tau_{y}}
$$

with $G_{\tau}(i, t)=G(i, t) X_{\tau}(i, t)$ 
Proof. Using the result presented in theorem (5.4) and the theorem (4.2) the proof is straightforward.

If the stopping times are defined in the original filtration, i.e., in the filtration $\mathcal{F}=\mathcal{F}_{0}, \mathcal{F}_{1}, \ldots, \mathcal{F}_{T}$, where $\mathcal{F}_{t}=\sigma\left(S_{t}\right)$ then randomized stopping times have to be considered.

Theorem 5.6 There is an adjusted probability measure $\bar{P} \in \overline{\mathcal{P}}\left(X_{y}\right)$ and a process $X \in \mathbb{X}$ such that the lower hedging price of an American derivative in a probabilistic dry market can be written as

$$
V_{p}^{u}=\max _{X \in \mathbb{X}} \max _{\bar{P} \in \overline{\mathcal{P}}\left(X_{y}\right)} E^{\bar{p}} G_{X}
$$

with $G_{X}(i, t)=G(i, t) X(i, t)$.

Proof. This result follows from the application of theorem (4.3) to the result presented in theorem (5.5).

\section{Comparison of the Results}

In this section we will compare the arbitrage-free bounds of an American derivative in a deterministic dry market, in a probabilistic dry market and in a market where transactions are possible at any point in time. In other words, we will compare the arbitrage-free bounds of an American derivative if, at some given points in time, transactions are not possible, transactions are possible with a given probability and transactions are possible.

The upper bound in a probabilistic dry market is higher than or equal to the upper bound if the market is dry in the deterministic sense. Moreover, it is also equal to or higher than the upper bound if transactions were possible at all points in time. The reason is that we are using the pure arbitrage-free concept. If, at a given point in time, it becomes possible to transact with a given probability, the seller of the American derivative must hedge against the possibility of exercise at that point in time. The value of the probability is irrelevant because he will hedge against the worse scenario. In what concerns the upper bound in a deterministic dry market it can be smaller or higher than the upper bound if transactions were possible at all points in time. The reason for this is quite intuitive. Consider an American derivative with a very high payoff in a given moment where transactions were not possible due to the deterministic dryness. If transactions were possible at that given moment in time, the value of the American derivative could increase to become higher than the upper bound in a deterministic dry market. 
The lower bound in a probabilistic dry market is higher than or equal to the lower bound if the market is dry in the deterministic sense and is equal to the lower bound if transactions were possible at all points in time $\left(V^{l}\right)$. In what concerns the comparison with the deterministic case note that the problem that must solved to obtain the lower bound in the deterministic case is a "subset" of the problem that is solved to find the lower bound in the probabilistic case. It corresponds to the $\mathbf{y}$ with the highest number of components equal to zero. Hence, $V_{p}^{l} \geq V_{d}^{l}$. On the other hand, the problem that must solved to obtain the lower bound in the case where transactions are possible at all points in time is also a "subset" of the problem that is solved to find the lower bound in the probabilistic case. It corresponds to the $\mathbf{y}$ with all components equal to one. Hence, $V_{p}^{l} \geq V^{l}$. Moreover, in the probabilistic case, the trading strategies that solve the problem for any $y$ whose components are not all equal to one is a possible solution when $y$ has all components equal to one. Therefore, we can conclude that $V_{p}^{l}=V^{l}$.

Considering that the only source of incompleteness in the market is the non-existence, or the possibility of non-existence, of the market at some points in time if transactions if transactions were possible at all points in time, markets would be complete and there would be a unique arbitrage-free value for any American derivative. We found out that this unique arbitragefree value for each American derivative is equal to the lower bound of the arbitrage-free range of variation for its value under a probabilistic dry market. However, it may not belong to the arbitrage-free range if a deterministic dry market is considered.

If the market is incomplete even with the existence of transactions at all points in time it is not possible to find a unique arbitrage free value for the American derivative. However, it is also possible to establish an arbitrage free range of variation for the value of the American derivative. This range will be a subset of the arbitrage free range of variation for the value of the American derivative in the case of probabilistic dryness, but may be wider than the arbitrage free range of variation in the deterministic case.

\section{$7 \quad$ Exercise Policy}

In order to understand the optimal exercise policy, we start presenting the case of a complete market. In this case, the value of an American derivative is given by

$$
V^{u}=\max _{\tau \in \mathbb{T}} \max _{P \in \mathcal{P}} E^{p} G_{\tau}
$$

where $G_{\tau}(i, t)=X_{\tau}(i, t) G(i, t)$, as before. 
If the solution is unique, the stopping time that solves (31) is the optimal exercise policy for the holder of the American derivative. The reason is as follows. Given an optimal stopping time $\tau^{*}$, we may define a stopping time frontier as the set of nodes $(i, t)$ such that $X_{\tau^{*}}(i, t)=1$. Recalling that there is an optimal stopping node for each possible path ${ }^{2}$, we define the interior of the stopping time frontier as the set of predecessors of the nodes that constitute the frontier. It follows that no rational agent exercises the American derivative at a node inside the stopping time frontier, because at such nodes, the American derivative is worth more than the corresponding exercise. Whenever the stopping time frontier is reached, the American derivative will be exercised by a rational agent. This happens because the derivative's payoff at that point is larger than the cost of a replicating portfolio, guaranteeing the derivative's payoff in the future.

If the solution is not unique there may be indeterminacy. An example illustrates this point. Consider the non-terminal node $\left(i, t_{1}\right)$ and the terminal nodes $\left(j, t_{2}\right)$ and $\left(m, t_{2}\right)$, which are the immediate successors of $\left(i, t_{1}\right)$. The replicating portfolio, at node $\left(i, t_{1}\right)$, is the pair $\left[\Delta\left(i, t_{1}\right), B\left(i, t_{1}\right)\right]$. Assume that this portfolio satisfies

$$
\begin{aligned}
\Delta\left(i, t_{1}\right) S\left(j, t_{2}\right)+B\left(i, t_{1}\right) R & =G\left(j, t_{2}\right) \\
\Delta\left(i, t_{1}\right) S\left(m, t_{2}\right)+B\left(i, t_{1}\right) R & =G\left(m, t_{2}\right)
\end{aligned}
$$

We also assume that, at node $\left(i, t_{1}\right)$,

$$
G\left(i, t_{1}\right)=V\left(i, t_{1}\right)
$$

In this case, the value of the portfolio, at node $\left(i, t_{1}\right)$, that replicates the value of the American derivative in nodes $\left(j, t_{2}\right)$ and $\left(m, t_{2}\right)$ is the same as the payoff of the American derivative. Let $P\left(i, t_{1}\right)$ denote the price of the American derivative at node $\left(i, t_{1}\right)$. In this case $P\left(i, t_{1}\right)=G\left(i, t_{1}\right)=V\left(i, t_{1}\right)$. Hence, the holder of the American derivative will obtain the same payoff exercising or selling the derivative.

In what concerns the dual variables, if, in the primal problem, the replicating portfolio satisfies 32 and 33 the solution of the dual problem is not unique. There are several node probability measures $q$ solving the maximization problem that characterizes the upper bound. Let $q_{1}$ and $q_{2}$ denote two possible solutions. In that case $q_{1}$ and $q_{2}$ must satisfy

$$
V^{u}=\max _{q_{1} \in Q} \sum_{\substack{(i, t) \in \mathcal{J}_{t} \\ t \in \mathcal{I}_{m}}} q_{1}(i, t) \bar{G}(i, t)=\max _{q_{2} \in Q} \sum_{\substack{(i, t) \in \mathcal{J}_{t} \\ t \in \mathcal{T}_{m}}} q_{2}(i, t) \bar{G}(i, t)
$$

\footnotetext{
${ }^{2}$ If the solution is unique, there is a unique strictly positive $q$ associated to each path. Hence, the stopping time is uniquely defined.
} 
such that for any $(i, t)$ with $t \in \mathcal{T}_{m}$

$$
\begin{aligned}
\sum_{m>t, m \in \mathcal{T}_{m}} \sum_{(j, m) \in i_{t}^{+}(m)} q_{1}(j, m)[\bar{S}(i, t)-\bar{S}(j, m)] & =0 \\
\sum_{m>t, m \in \mathcal{T}_{m}} \sum_{(j, m) \in i_{t}^{+}(m)} q_{2}(j, m)[\bar{S}(i, t)-\bar{S}(j, m)] & =0 .
\end{aligned}
$$

If the maximization problem characterizing the upper bound is not uniquely solved by a node probability measure $q$, then the stopping time and the adjusted probability measure are also not uniquely defined. For instance, the upper bound can be written as

$$
V^{u}=\max _{\tau_{1} \in \mathbb{T}} \max _{P_{1} \in \mathcal{P}\left(\tau_{1}\right)} E^{p_{1}} G_{\tau_{1}}=\max _{\tau_{2} \in \mathbb{T}} \max _{P_{2} \in \mathcal{P}\left(\tau_{2}\right)} E^{p_{2}} G_{\tau_{2}}
$$

with $X_{\tau_{1}}\left(i, t_{1}\right)=1, X_{\tau_{1}}\left(j, t_{2}\right)=X_{\tau_{1}}\left(m, t_{2}\right)=0, X_{\tau_{2}}\left(i, t_{1}\right)=0$ and $X_{\tau_{2}}\left(j, t_{2}\right)=X_{\tau_{2}}\left(m, t_{2}\right)=1$. Actually, the solution can be written with randomized stopping times. See the appendix for an example.

As the stopping time is not unique there are several stopping time frontiers, each one associated with a different stopping time. For any node inside all stopping time frontiers, the argument of the unique case solution applies. The agent does not have an incentive to exercise the American derivative. However, when the first stopping time frontier is reached, namely node $\left(i, t_{1}\right)$, the American derivative may be exercised. At node $\left(i, t_{1}\right)$ the value of the replicating portfolio, the payoff of the American derivative and the market value are the same. If the holder of the American derivative wants to guarantee the highest possible payoff at node $\left(i, t_{1}\right)$ he must exercise or sell the derivative. If the holder of the American derivative wants to guarantee a given payoff at some successor of $\left(i, t_{1}\right)$, then he may have an incentive to exercise, or sell the American derivative, and buy a replicating portfolio ${ }^{3}$. Hence, by exercising the American derivative the agent can assure a better result (although not strictly better) than by selling, as in the unique case solution. If the American derivative is not exercised at any of the stopping time frontiers, when the last one is reached the American derivative will be exercised, for the reasons presented in the unique case solution.

With incomplete markets the problem is more complex. In order to characterize the optimal exercise policy we use the stopping time $\left(\tau_{*}^{u}\right)$ that is solution of the problem characterizing the upper bound of the arbitrage free range of variation. Several points must be addressed. First, if the reduced

\footnotetext{
${ }^{3}$ Note that this portfolio has the same payoff as the American derivative at some successors, and a higher payoff than the American derivative at other successors.
} 
filtration is considered, the solution may involve randomized stopping times. Hence, it is not possible to conclude about an optimal exercise policy.

Second, if we consider the enlarged filtration, where ordinary stopping times are enough to describe the upper bound, the stopping time is not uniquely defined for all paths. Even for all paths with a strictly positive $q$ the optimum stopping time, $\tau_{*}^{u}$, is not uniquely defined. Let $(j, m)$ be the node such that $q(j, m)>0$. Hence, the associated process $X_{\tau_{*}^{u}}(.,$.$) will be equal to$ one at that node, i.e., $X_{\tau_{*}^{u}}(j, m)=1$. If node $(j . m)$ is reached the derivative will be exercised. However, it may be exercised at any predecessor of node $(j, m)$. The reason is as follows. Using pure arbitrage arguments it is possible to conclude that at any predecessor of $(j, m)$ the price of the derivative is higher, or equal, to its payoff. Let a predecessor of $(j, m)$ be denoted by $(k, n)$. If the price is higher than the payoff, i.e., $P(k, n)>G(k, n)$, any rational agent is better selling rather than exercising the derivative. If the price of the derivative is equal to its payoff, i.e., $P(k, n)=G(k, n),{ }^{4}$ a rational holder who wants to guarantee a given amount at that point in time is indifferent between exercising or selling the derivative ${ }^{5}$. However, if the American derivative was not exercised at any node that is a predecessor of the stopping time frontier it will be exercised when the stopping time frontier is reached. The reason is that the payoff is higher than the price and the value of the replicating portfolio. However, if in a given path there are not any node with a strictly positive $q$, the optimum stopping time can be such that $X_{\tau_{*}^{u}}(.,$.$) is one in any$ node that has zero probability measure. As it is possible to have more than one node with zero probability measure the exercise policy is not uniquely defined.

Third and final point, the same problem that occurs in the complete market case when the solution of the dual problem is not unique happens when market are incomplete.

\section{Conclusion}

We have shown that in the case of deterministic illiquidity the bounds for the values of American derivatives are the supremum of the implied European derivatives, this supremum being taken over deterministic stopping times. In

\footnotetext{
${ }^{4}$ In this case $V^{l}(k, n)=G(k, n)<V^{u}(k, n)$

${ }^{5}$ If a rational agent wants to guarantee a given amount at any predecessor of $(j, m)$ he may have an incentive to sell, or exercise, the derivative and buy the superreplicating portfolio. In spite the value of this portflio is higher than the amount received from selling, or exercising, the derivative it has a higher payoff in the future.
} 
the probabilistic case there is an additional source of uncertainty, the existence or not of the market at given points in time, which can be interpreted as the realization of an additional stochastic process. If an enlarged filtration, resulting from the price process and the market existence process is considered, only ordinary stopping times are required to describe the upper and lower bounds. However, if the enlarged filtration were not considered, and the stopping times were defined using only the filtration induced by the price process, then they could be randomized, just as in Jha and Chalasani (2001).

The point that explains the difference between our result and that of Jha and Chalasani (2001) is the following. In their paper, rebalancing the superhedging portfolio is possible at any point in time, and the derivatives have well defined payoffs at any point in time. However, due to transaction costs, it may be optimal for their problem not to rebalance at some points in time. The cheapest superhedging strategy could then be to replicate the derivative's payoff in consecutive points in time, for a given path. These points with full replication correspond to optimal stopping. Since there may be more than one per path, the optimal stopping time would be randomized. In our case it is not possible to exercise the derivative when there is no market for the underlying asset, and hence there is no need to hedge for exercise at those points where it is not possible to rebalance the portfolio. In particular, in the case of probabilistic illiquidity, our representation of the superreplicating bounds with deterministic stopping times is strongly driven by the fact that we consider the enlarged filtration resulting from the price process and the market-existence process. If that were not case, the resulting stopping times could also be randomized. In fact, had we considered only the filtration generated by the price process, for any given price path it would be optimal to hedge the payoff at different points in time.

In a complete market the arbitrage free value of the derivative is unique and equal to the value of the replicating portfolio. Moreover, when an American derivative is considered under such conditions, the optimal exercise policy corresponds to the stopping time that is the supremum of the implied European derivatives. However, in an incomplete market framework, the superreplication methodology results in an arbitrage-free range of variation for the value of the derivative. Hence, the market value of the derivative is not defined under the strict absence of arbitrage. Consistently, if American options are considered in this setting, the optimal exercise policy is also not well defined. The reason is that are paths were the stopping time is not uniquely defined and, in addition, if the filtration induced by the price process is considered, randomized stopping time must be used.

More technically the argument translates into the following. Under no 
transaction costs and complete markets, there is only one node per path such that the value of the superhedging portfolio fully replicates the derivative's payoff. This unique node per path would correspond to the optimal exercise of the derivative. Under market incompleteness, however, there are paths for which such nodes do not exist, therefore not allowing a unique definition of the stopping time. In the setting of Jha and Chalasani (2001), in particular, there may be some other paths for which more than one such nodes exist, generating the randomness of the stopping times corresponding to those paths. When dealing with dry markets, use of the enlarged filtration described above allows for at most one such node per path, avoiding in this way the randomized stopping times. Hence, arbitrage arguments are not enough to define the optimal exercise policy.

These conclusions are valid under the assumption that there exists a unique solution for the dual problem when determining the upper bound, as it is generally the case in both our setting and Jha and Chalasani's. If there is more than one solution for the dual problem with respect to a given path, then there will exist more than one node satisfying full replication of the derivative's payoff for that path, even in complete markets. Moreover, one of the solutions of the problem could always be written in terms of randomized stopping times. However, both in complete markets and in dry markets under the enlarged filtration, it is possible to write a solution in terms of ordinary stopping times.

There are several pricing alternatives in the literature to characterize the market value, or simply to restrict the arbitrage free range of variation. The different approaches ${ }^{6}$ used with European options to choose a value in the arbitrage-free range, or to restrict that range, could be helpful with American derivatives. Notice that an important drawback of the bounds obtained with probabilistic illiquidity is that these bounds do not depend on the probability of market existence. However, if a statistical arbitrage concept, in the spirit of Bondarenko (2002), rather than pure arbitrage is used, the bounds could depend on the probability of the existence of the market. This is a further line of research to be pursued.

\footnotetext{
${ }^{6}$ Equilibrium or utility based approach, as in Rubinstein (1976), Davis (1997) ; risk/reward criterion as in Bernardo and Ledoit (2000), Cochrane and Saá-Requejo (2000) and Bondarenko (2002); and considering the market price of risk associated with non traded state variables as in Heston (1993).
} 


\section{References}

[1] Amaro de Matos, J. and P. Antão, 2001, "Super-Replicating Bounds on European Option Prices when the Underlying Asset is Illiquid", Economics Bulletin, 7, 1-7.

[2] Bensoussan, A., 1984, "On the Theory of Option Pricing", Acta Applicandae Mathematicae 2, 139-158.

[3] Bernardo, A. and O. Ledoit, 2000, "Gain, Loss and Asset Pricing", Journal of Political Economy, 108, 144-172.

[4] Bondarenko, O., 2003, "Statistical Arbitrage and Securities Prices", The Review of Financial Studies, 16, 3, 873-919.

[5] Cochrane, J.H. and J. Saá-Requejo, 2000, "Beyond Arbitrage: GoodDeal Asset Price Bounds in Incomplete Markets", Journal of Political Economy, 108, 79-119.

[6] Davis, M. H. A., 1997, "Option Pricing in Incomplete Markets", in Mathematics of Derivative Securities, M. A. H. Dempster and S. R Pliska (eds.), Cambridge University Press, 216-226.

[7] Chalasani, P. and Jha,S., 2001, "Randomized Stopping Times and American Option Pricing with transaction costs", Mathematical Finance, 11-1, 33-77.

[8] Edirisinghe, C., Naik, V. and R. Uppal, 1993, "Optimal Replication of Options with Transactions Costs and Trading Restrictions", Journal of Financial and Quantitative Analysis, 28, 117-138.

[9] El Karoui, N. and Quenez, M.C., 1991, "Programation dynamique et évaluation des actifs contingents en marché incomplet", Comptes Rendues de l'Academy des Sciences de Paris, Série I, 313, 851-854.

[10] El Karoui, N. and Quenez, M.C., 1995, "Dynamic Programming and Pricing of Contingent Claims in an Incomplete Market", SIAM Journal of Control and Optimization, 33, 29-66.

[11] Follmer, H. and Y. Kabanov, 1998, "Optional Decomposition and Lagrange Multipliers", Finance 83 Stochastics 2, 69-81.

[12] Follmer, H. and D. Kramkov, 1997, "Optional Decomposition under Constraints", Probability Theory and Related Fields 109, 1-25. 
[13] Harrison, J. and Kreps, D., 1979, "Martingales and Arbitrage in a Multiperiod Securities Markets", Journal of Economic Theory 20, 381-408.

[14] Heston, S. , 1993, "A Closed-Form Solution for Options with Stochastic Volatility with Applications to Bond and Currency Options", Review of Financial Studies, 6, 327-344.

[15] Karatzas, I., 1988, "On the Pricing of American Options", Applied Mathematics and Optimization 17, 37-60.

[16] Karatzas, I. and S. Kou, 1996, "On the Pricing of Contingent Claims with Constrained Portfolios", The Annals of Applied Probability 6, 321369.

[17] Karatzas, I. and S. Kou, 1998, "Hedging American Contingent Claims with Constrained Portfolios", Finance 83 Stochastics 3, 215-258.

[18] Kramkov, D., 1996, "Optional Decomposition of Supermartigales and Hedging Contingent Claims in Incomplete Security Markets", The Annals of Applied Probability 9, 904-950.

[19] Longsatff, F., 2001, "Optimal Portfolio Choice and the Valuation of Illiquid Securities", Review of Financial Studies, 14, 407-431.

[20] Rubinstein, M., 1976, "The Valuation of Uncertain Income Streams and the Pricing of Options", Bell Journal of Economics and Management Science, 7, 407-425.

\section{A Proof of theorems 2.1 and 2.2 of section 2.2}

\section{A.1 Proof of theorem 2.2}

Proof. This theorem is corollary 5.5 of Jha and Chalasani (2001) with nodes corresponding to trading dates $t \in \mathcal{T}_{m}$. So, the proof of theorem 2.2 follows the one presented there. In order to proof that for a given measure strategypair $\left(P, X_{\tau}\right)$ the simple node measure $q(i, t)=P(i, t) X_{\tau}(i, t)$ is the unique 
simple node measure we only need to check that $q(i, t) \geq 0$ and

$$
\begin{aligned}
& \sum_{t \in \mathcal{T}_{m}} \sum_{(j, t) \in \mathcal{J}_{t}} q(j, t) \\
= & \sum_{t \in \mathcal{T}_{m}} \sum_{(j, t) \in \mathcal{J}_{t}} P(i, t) X_{\tau}(i, t) \\
= & \sum_{(i, T) \in \mathcal{J}_{T}} P(i, T) \sum_{(j, t) \in w \supset(i, T)} X_{\tau}(j, t)=1 .
\end{aligned}
$$

On the other hand, for any give node $(i, t)$ such that

$$
q(i, t)+\sum_{j \in i^{+}(\tau)} \sum_{\substack{\tau>t \\ \tau \in \mathcal{T}_{m}}} q(j, \tau)>0
$$

the adjusted probability measure is uniquely defined and given by

$$
P(i, t)=q(i, t)+\sum_{j \in i^{+}(\tau)} \sum_{\substack{\tau>t \\ \tau \in \mathcal{T}_{m}}} q(j, \tau) .
$$

For all other nodes the adjusted probability measure is defined forwardinductively in the following way. Consider any non-terminal node $\left(i, t_{1}\right)$ with $q\left(i, t_{1}\right)>0$. Then, for a given immediate successor of $\left(i, t_{1}\right)$, denoted $\left(j, t_{2}\right)$, set $P\left(j, t_{2}\right)=P\left(i, t_{1}\right)$. For all other immediate successors of $\left(i, t_{1}\right)$ set the adjusted probability equal to zero. If $\left(j, t_{2}\right)$ is a nonterminal node then same process applies until a terminal node is reached.

In what concerns the adapted process $X_{\tau}$ it is defined as follows

$$
X_{\tau}(i, t)= \begin{cases}q(i, t) & , \quad P(i, t)>0 \\ \sum_{j \in i_{t_{2}}^{+}(\tau)} \sum_{\substack{\tau>t_{2} \\ \tau \in \mathcal{T}_{m}}} q(j, \tau) & P(i, t)=0 \text { and } \\ 0 & P\left(j, t_{2}\right)>0 \text { with }\left(j, t_{2}\right) \in i_{t}^{-}\end{cases}
$$

The argument to proof the uniqueness of $X_{\tau}(i, t)$ when $q(i, t)$ is strictly positive is as follows. As $X_{\tau}(i, t)$ is equal to one or to zero when $q(i, t)$ is strictly positive then $X_{\tau}(i, t)$ is equal to one. On the other hand, if $q(i, t)>$ 0 and $X_{\tau}(i, t)=1$ in order to have $q(i, t)=p(i, t) X_{\tau}(i, t)$ the adjusted probability measure $p(i, t)$ has to be equal to $q(i, t)$. Additionally, as the adjusted probability at any given point is the sum of the adjusted probability on all successors the adjusted probability measure is uniquely defined in all nodes such that

$$
q(i, t)+\sum_{j \in i^{+}(\tau)} \sum_{\substack{\tau>t \\ \tau \in \mathcal{T}_{m}}} q(j, \tau)>0
$$




\section{A.2 Proof of theorem 2.1}

Proof. Consider some nonsimple node-measure $q \in Q$. We will construct two new node probability measures $a$ and $c$ that belong to $Q$ such that $q=\frac{1}{2}(a+c)$. Hence, as any nonsimple node-measure is not an extreme point we can conclude that an extreme point has to be a simple node-measure.

If $q$ is a nonsimple node-measure then there is a node $(i, t)$ such that $q(i, t)>0$ and $\sum_{\tau \in \mathcal{T}_{m}} \sum_{(j, \tau) \mathbf{i}_{t}^{+}(\tau)} q(j, \tau)>0$. Consider such a node $(i, t)$. Fix some strictly positive $\varepsilon$ such that $\varepsilon<q(i, t)$ and for any $q(j, k)>0$, with $k \in \mathcal{T}_{m}, k>t$ and $(j, k) \in i_{t}^{+}(k)$, we have $\varepsilon<q(j, k)$. Define a node measure $a$ that is identical to $q$ everywhere except that

$$
a(i, t)=q(i, t)-\varepsilon
$$

and, for any $(j, k)$ such that $q(j, k)>0$ with $k \in \mathcal{T}_{m}, k>t$ and $(j, k) \in$ $i_{t}^{+}(k)$,

$$
a(j, k)=q(j, k)\left(1+\frac{\varepsilon}{\sum_{(j, \tau) \in \mathbf{i}_{t}^{+}(\tau)} \sum_{\substack{\tau>t \\ \tau \in \mathcal{T}_{m}}} q(j, \tau)}\right) .
$$

Note that the total amount by which $q$ is increased on all the successors of $(i, t)$ matches the amount by which $q$ is decreased at $(i, t)$-that is, a is just a redistribution of $q$, and so is also a node-measure. The above statement also hold for the node-function c constructed as a but with $-\varepsilon$ instead of $\varepsilon$. It is easy to see that $q=\frac{1}{2}(a+c)$.

In order to conclude that $a \in Q$ we need to check that the following conditions hold

$$
\begin{gathered}
a(j, t) \geq 0, \\
\sum_{t \in \mathcal{T}_{m}} \sum_{(j, t) \in \mathcal{J}_{t}} a(j, t)=1
\end{gathered}
$$

and

$$
\sum_{\substack{\tau>t^{\prime} \\ \tau \in \mathcal{T}_{m}}} \sum_{(j, \tau) \in \mathbf{i}_{t^{\prime}}^{+}(\tau)} a(j, \tau) \bar{S}\left(k, t^{\prime}\right)=\sum_{\substack{\tau>t^{\prime} \\ \tau \in \mathcal{T}_{m}}} \sum_{(j, \tau) \in \mathbf{i}_{t^{\prime}}^{+}(\tau)} a(j, \tau) \bar{S}(j, \tau) .
$$

The restrictions of equations (35) and (34) are trivially respected. In what concerns the restriction in equation (36) only the relevant path is being 
analyzed. The restriction presented in equation (36) can be written as

$$
\begin{aligned}
& \sum_{\substack{\tau>t^{\prime} \\
\tau \in T_{m}}} \sum_{(j, \tau) \in \mathbf{i}_{t^{\prime}}^{+}(\tau)}[q(j, \tau)+\sigma(j, \tau)] \bar{S}\left(k, t^{\prime}\right) \\
= & \sum_{\substack{\tau>t^{\prime} \\
\tau \in \mathcal{T}_{m}}} \sum_{(j, \tau) \in \mathrm{i}_{t^{\prime}}^{+}(\tau)}[q(j, \tau)+\sigma(j, \tau)] \bar{S}(j, \tau)
\end{aligned}
$$

where $\sigma(j, \tau)$ defined as

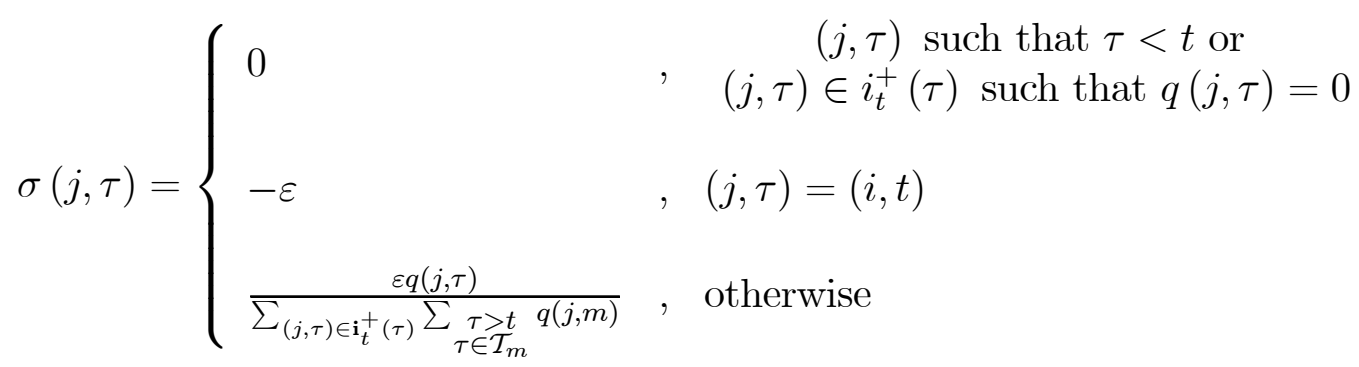

As

$$
\sum_{\substack{\tau>t^{\prime} \\ \tau \in \mathcal{I}_{m}}} \sum_{(j, \tau) \in \mathbf{i}_{t^{\prime}}(\tau)} q(j, \tau) \bar{S}\left(k, t^{\prime}\right)=\sum_{\substack{\tau>t^{\prime} \\ \tau \in \mathcal{I}_{m}}} \sum_{(j, \tau) \in \mathbf{i}_{t^{\prime}}^{+}(\tau)} q(j, \tau) \bar{S}(j, \tau)
$$

equation (37) can be written as

$$
\sum_{\substack{\tau>t^{\prime} \\ \tau \in \mathcal{T}_{m}}} \sum_{(j, \tau) \in \mathbf{i}_{t^{\prime}}^{+}(\tau)} \sigma(j, \tau) \bar{S}\left(k, t^{\prime}\right)=\sum_{\substack{\tau>t^{\prime} \\ \tau \in \mathcal{T}_{m}}} \sum_{(j, \tau) \in \mathbf{i}_{t^{\prime}}^{+}(\tau)} \sigma(j, \tau) \bar{S}(j, \tau) .
$$

In order to check that equation (38) holds two alternative situations will be considered. The first one is $t^{\prime} \leq t$. In this case, equation (38) can be written as

$$
\begin{aligned}
& \varepsilon \bar{S}\left(k, t^{\prime}\right)+\frac{\varepsilon}{\sum_{j \in i^{+}(m)} \sum_{\substack{m>t \\
m \in \mathcal{T}_{m}}} q(j, m)} \sum_{j \in i(\tau)} \sum_{\substack{\tau>t \\
\tau \in \mathcal{T}_{m}}} q(j, \tau) \bar{S}\left(k, t^{\prime}\right) \\
= & \varepsilon \bar{S}(i, t)+\frac{\varepsilon}{\sum_{j \in i^{+}(m)} \sum_{\substack{m>t \\
m \in \mathcal{T}_{m}}} q(j, m)} \sum_{j \in i(\tau)} \sum_{\substack{\tau>t \\
\tau \in \mathcal{T}_{m}}} q(j, \tau) \bar{S}(j, \tau) .
\end{aligned}
$$

As both members are equal to zero the equality holds. The second case to be considered is $t^{\prime}>t$. In this case, equation (38) can be written as

$$
\begin{aligned}
& \frac{\varepsilon}{\sum_{j \in i(\tau)} \sum_{\substack{\tau>t \\
\tau \in \mathcal{T}_{m}}} q(j, \tau)} \sum_{j \in i(\tau)} \sum_{\substack{\tau>t^{\prime} \\
\tau \in \mathcal{T}_{m}}} q(j, \tau) \bar{S}\left(k, t^{\prime}\right) \\
= & \frac{\varepsilon}{\sum_{j \in i(\tau)} \sum_{\substack{\tau>t \\
\tau \in \mathcal{T}_{m}}} q(j, \tau)} \sum_{j \in i(\tau)} \sum_{\substack{\tau>t^{\prime} \\
\tau \in \mathcal{T}_{m}}} q(j, \tau) \bar{S}(j, \tau)
\end{aligned}
$$

As in the previous case, both members are equal to zero and the equality holds. 


\section{B Proof of theorem 4.1, 4.2 and 4.3 of section 4.2}

\section{B.1 Proof of theorem 4.1}

Proof. Consider that the auxiliary node probability measures $\hat{q}(i, t ; \mathbf{y})$ which, for any $\mathbf{y}$, is defined for all $t \in \mathcal{T}_{\mathbf{y}}$ and $t \in \max \left(t \in \mathcal{T}_{p}: y_{t}=0\right)$. By construction $\hat{q}(i, t ; \mathbf{y})$ is equal to zero for $t \in \max \left(t \in \mathcal{T}_{p}: y_{t}=0\right)$ and $\hat{q}(i, t ; \mathbf{y})=q(i, t ; \mathbf{y})$ for all $t \in \mathcal{T}_{y}$. Applying theorem 2.1 to the node probability measure $\hat{q}(i, t ; \mathbf{y})$ theorem 4.1 is straightforward.

\section{B.2 Proof of theorem 4.2}

Proof. Consider that the auxiliary node probability measures , $\tilde{q}(i, t ; \mathbf{y})$, and the auxiliary adjusted probability measures, $\tilde{p}(i, t ; \mathbf{y})$ and $\tilde{X}_{\tau}(i, t ; \mathbf{y})$ are also defined for all $t \in \max \left(t \in \mathcal{T}_{p}: y_{t}=0\right)$. By construction, and $\tilde{q}(i, t ; \mathbf{y})$ is equal to zero for all $t \in \max \left(t \in \mathcal{T}_{p}: y_{t}=0\right)$ and $\tilde{q}(i, t ; \mathbf{y})=q(i, t ; \mathbf{y})$ for all $t \in \mathcal{T}_{y}$.

Applying theorem 2.2 we find that

$$
\tilde{p}(i, t ; \mathbf{y})=\sum_{\left\{\mathbf{z}: z_{0}=y_{0}, \ldots, z_{t}=y_{t}\right\}} \sum_{(j, t+1)} \tilde{p}(j, t+1 ; \mathbf{z}) .
$$

and $\tilde{X}_{\tau}(i, t ; \mathbf{y})=0$, for all $t \in \max \left(t \in \mathcal{T}_{p}: y_{t}=0\right)$.

\section{B.3 Proof of theorem 4.3}

Proof. The proof that $\bar{P}(i, t)$ is a adjusted probability measure is straightforward. In what concerns the randomized stopping time, $X$, we must check that $X(i, t) \geq 0$ and condition (17) is satisfied.

For any given node $(j, t+1) \in i_{t}^{+} \operatorname{such} \alpha(j, t+1) \neq 0$ we have

$$
\begin{aligned}
X(i, t) & =\frac{\alpha(i, t)}{\bar{P}(i, t)}-\frac{\alpha(i, t)-\sum_{\left\{\mathbf{y}: y_{t}=1\right\}} p(i, t ; \mathbf{y}) X_{\tau}(i, t ; \mathbf{y})}{\bar{P}(i, t)} \\
& =\frac{\alpha(i, t)}{\bar{P}(i, t)}-\frac{\sum_{(j, t+1) \in i_{t}^{+}} \alpha(j, t+1)}{\bar{P}(i, t)} \\
& =\frac{\alpha(i, t)}{\bar{P}(i, t)}-\frac{\alpha(j, t+1)}{\bar{P}(j, t+1)} .
\end{aligned}
$$


Consider a given path such that at the terminal node $(i, T)$ we have $\alpha(i, T)>$ 0 . In that case

$$
\sum_{(i, t) \in w} X(i, t)=1
$$

Consider a given path such that at the node $(j, s)$ we have $\alpha(i, t) \neq 0$ and $\alpha(m, t+1)=0$. In that case

$$
\sum_{(k, r) \in w} X(k, r)=1-\frac{\alpha(i, t)}{\bar{P}(i, t)}+X(i, t)+X(m, t+1) .
$$

Let $(h, t+1)$ be a successor of $(i, t)$. If $\alpha(h, t+1) \neq 0$ then $\bar{P}(m, t+1)=0$. Moreover, as $\bar{P}(i, t) \neq 0$ then $X(m, t+1)=\frac{\sum_{(i, t+1) \in i_{t}^{+}(t+1)} \alpha(i, t+1)}{P(i, t)}$. As a result, equation (39) can be written as

$$
\begin{aligned}
\sum_{(k, r) \in w} X(k, r)= & 1-\frac{\alpha(i, t)}{\bar{P}(i, t)}+\frac{\sum_{\left\{\mathbf{y}: y_{t}=1\right\}} p(i, t ; \mathbf{y}) X_{\tau}(i, t ; \mathbf{y})}{\bar{P}(i, t)} \\
& +\frac{\sum_{(i, t+1) \in i_{t}^{+}(t+1)} \alpha(i, t+1)}{\bar{P}(i, t)} \\
= & 1 .
\end{aligned}
$$

However, if there are not a successor $(h, t+1)$ of $(i, t)$ such that $\alpha(h, t+1) \neq$ 0 then

$$
\begin{aligned}
\sum_{(k, r) \in w} X(k, r)= & 1-\frac{\alpha(i, t)}{\bar{P}(i, t)}+X(i, t)+X(m, t+1) \\
& +\sum_{\substack{(k, r) \in w \\
r \geq t+1}} X(k, r) .
\end{aligned}
$$

$\bar{P}(m, t+1)$ can take two possible values: 0 and $\bar{P}(i, t)$. Let us consider the two possibilities:

- $\bar{P}(m, t+1)=0$. This situation is the same as the one just described.

- $\bar{P}(m, t+1)=\bar{P}(i, t)$. As $\alpha(i, t)=\sum_{\left\{\mathbf{y}: y_{t}=1\right\}} p(i, t ; \mathbf{y}) X_{\tau}(i, t ; \mathbf{y})$ then $-\frac{\alpha(i, t)}{P(i, t)}+X(i, t)=0$. Moreover, $X(m, t+1)=0$. For any $(k, r) \in m_{t+1}^{+}(r)$ such that $\bar{P}(k, r)=\bar{P}(m, t+1)$ then $X(k, r)=0$. For a given $(k, r) \in m_{t+1}^{+}(r)$ such that $\bar{P}(k, r)=0$ and $\bar{P}(i, r-1)$ with $(k, r) \in i_{r-1}^{+}$then $X(k, r)=$ $\frac{\sum_{(k, r) \in i_{t}^{+}(r)} \alpha(k, r)}{P(m, t+1)}=0$

As a result, equation (40) is verified and the proof is complete. 


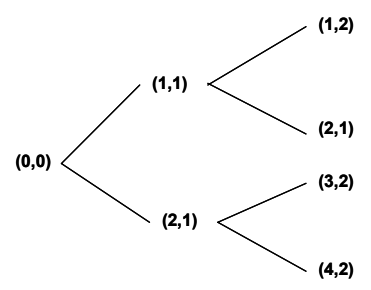

Figure 4:

\section{Exercise Policy}

Example C.1 Consider a binomial tree with two periods. This framework is described in the following way where in parenthesis are identified the nodesThe primal problem can be written as

$$
V^{u}=\min _{\{\Delta(0,0), B(0,0), \Delta(1,1), B(1,1), \Delta(2,1), B(2,1)\}} \Delta(0,0) S(0,0)+B(0,0)
$$

subject to the following conditions

$$
\begin{aligned}
& \Delta(0,0) S(0,0)+B(0,0) \geq G(0,0), \\
& \Delta(0,0) S(1,1)+B(0,0) R \geq G(1,1), \\
& \Delta(0,0) S(2,1)+B(0,0) R \geq G(2,1), \\
& \Delta(1,1) S(1,2)+B(1,1) R=G(1,2), \\
& \Delta(1,1) S(1,2)+B(1,1) R=G(1,2), \\
& \Delta(2,1) S(3,2)+B(2,1) R=G(3,2), \\
& \Delta(2,1) S(4,2)+B(2,1) R=G(4,2),
\end{aligned}
$$

and the self-financing restrictions

$$
\begin{aligned}
& \Delta(0,0) S(1,1)+B(0,0) R \geq \Delta(1,1) S(1,1)+B(1,1) \\
& \Delta(0,0) S(2,1)+B(0,0) R \geq \Delta(2,1) S(2,1)+B(2,1) .
\end{aligned}
$$


The dual problem can be written as

$$
\begin{aligned}
& \max _{q(0,0)} q(0,0) \bar{G}(0,0)+\sum_{i=1}^{2} q(i, 1) \bar{G}(i, t)+\sum_{i=1}^{4} q(i, 2) \bar{G}(i, t) \\
& \{q(i, 1)\}_{i=1,2} \\
& \{q(j, 2)\}_{j=1,2,3,4}
\end{aligned}
$$

such that

$$
\begin{aligned}
& \sum_{i=1}^{2} q(i, 2) S(1,1)=\sum_{i=1}^{2} q(i, 2) \bar{S}(i, 2) \\
& \sum_{i=3}^{4} q(i, 2) S(2,1)=\sum_{i=3}^{4} q(i, 2) \bar{S}(i, 2)
\end{aligned}
$$

and

$$
\left[\sum_{i=1}^{4} q(i, 2)+\sum_{i=1}^{2} q(i, 1)\right] S(0,0)=\sum_{i=1}^{4} q(i, 2) \bar{S}(i, 2)+\sum_{i=1}^{2} q(i, 1) \bar{S}(i, 1)
$$

Consider the case where the interest rate is zero and the value of the underlying asset and the payoffs of the American derivative can be described as $A$

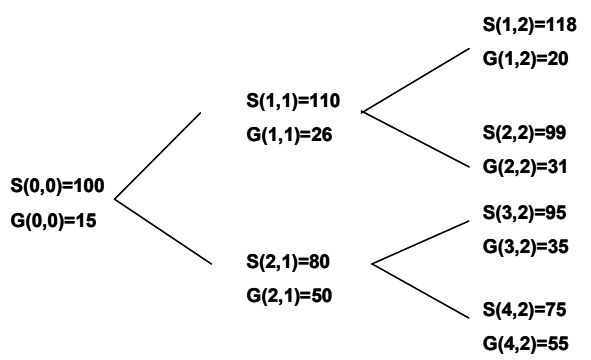

Figure 5: 
possible ${ }^{789}$ solution of the primal problem is

$$
\begin{array}{ccc} 
& \Delta(., .) & B(., .) \\
(0,0) & -0.8 & 114 \\
(1,1) & -0,579 & 88,32 \\
(2,1) & -1 & 130
\end{array}
$$

Two possible solutions of the dual problem are given by

$\begin{array}{cccc} & q^{1}(., .) & q^{2}(., .) & q^{3}(., .) \\ (0,0) & 0 & 0 & 0 \\ (1,1) & 0,6667 & 0,6667 & 0,6667 \\ (2,1) & 0,3333 & 0 & 0,2933 \\ (1,2) & 0 & 0 & 0 \\ (1,2) & 0 & 0 & 0 \\ (1,2) & 0 & 0,0833 & 0,01 \\ (1,2) & 0 & 0,25 & 0,03\end{array}$

The correspondent stopping time and probability measures ${ }^{10}$ are given in the next table. The probability measure $P \in \mathcal{P}$, that is uniquely defined, is

\footnotetext{
${ }^{7}$ The portfolio $\Delta(0,0)$ and $B(0,0)$ is uniquely defined because it is the only one that replicates at time $t=1$. At node node $(1,1)$ the value of the portfolio $[\Delta(0,0), B(0,0)]$ is equal to $G(1,1)$ because the value of the portfolio that replicates $G(1,2)$ and $G(2,2)$ is smaller than $G(1,1)$. On the other hand, at node $(2,1)$ the value of the portfolio $[\Delta(0,0), B(0,0)]$ is equal to $G(2,1)$ and to value of the replicating portfolio at $t=2$, because they coincide. Any other portfolio that superreplicates at time $t=1$ would be more expensive.

${ }^{8}$ The portolio $\Delta(1,1)$ and $B(1,1)$ is not uniquely defined because the value at node $(1,1)$ of the porfolio that replicates the payoffs of the American derivative at nodes $(1,2)$ and $(2,2)$ is smaller that $G(1,1)$.

${ }^{9}$ In node $(2,1)$ the value of the portfolio that replicates the payoff of the American derivative at nodes $(3,2)$ and $(4,2)$ is equal to the payoff of the American derivative, i.e., $\Delta(2,1) S(2,1)+B(2,1)=G(2,1)$ Hence, any other portfolio that replicates the payoffs will have a cost higher than $G(2,1)$ resulting in a higher value of the function. As a result, the portfolio in node $(2,1)$ is unique.

${ }^{10}$ The probability measures are not uniquely defined. The probability in bold menas that it is uniquely defined in these nodes.
} 
presented in the last row.

$\begin{array}{cccccccc}(0,0) & 0 & \mathbf{1} & 0 & \mathbf{1} & 0 & \mathbf{1} & 1 \\ (1,1) & 1 & \frac{\mathbf{2}}{3} & 1 & \frac{\mathbf{2}}{3} & 1 & \frac{\mathbf{2}}{\mathbf{3}} & \frac{\mathbf{2}}{\mathbf{3}} \\ (2,1) & 1 & \frac{1}{3} & 0 & \frac{1}{3} & 0.88 & \frac{1}{3} & \frac{1}{\mathbf{3}} \\ (1,2) & 0 & 0 & 0 & \frac{2}{3} & 0 & 0 & \frac{22}{57} \\ (2,2) & 0 & \frac{2}{3} & 0 & 0 & 0 & \frac{2}{3} & \frac{16}{57} \\ (3,2) & 0 & 0 & 1 & \frac{1}{12} & 0.12 & \frac{1}{12} & \frac{1}{12} \\ (4,2) & 0 & \frac{1}{3} & 1 & \frac{1}{4} & 0.12 & \frac{1}{4} & \frac{1}{4}\end{array}$

If assumption 1 holds the exercise policy can be given by the stopping time $\tau^{1}$ or $\tau^{2}$. However, if assumption 2 holds the exercise policy is given by stopping time $\tau^{2}$. 